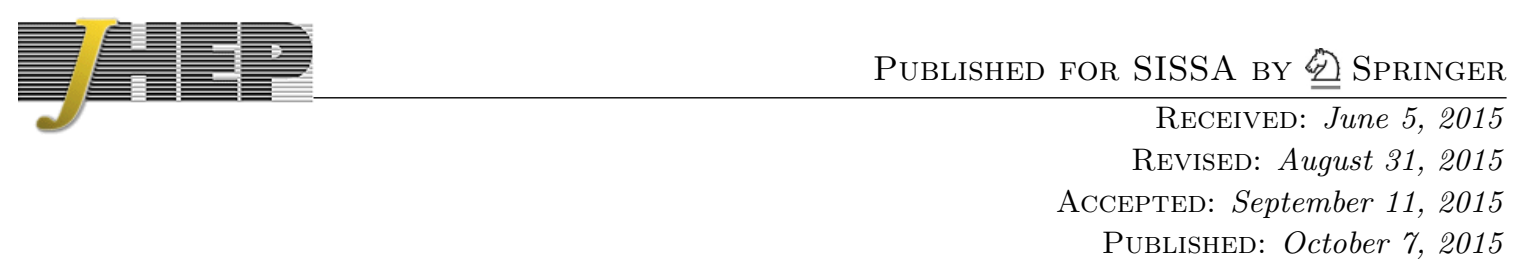

\title{
Study of lepton flavor violation in flavor symmetric models for lepton sector
}

\author{
Tatsuo Kobayashi, ${ }^{a}$ Yuji Omura, ${ }^{b}$ Fumihiro Takayama $^{c}$ and Daiki Yasuhara $^{d}$ \\ ${ }^{a}$ Department of Physics, Hokkaido University, \\ Sapporo 060-0810, Japan \\ ${ }^{b}$ Kobayashi-Maskawa Institute for the Origin of Particles and the Universe (KMI), \\ Nagoya University, \\ Nagoya 464-8602, Japan \\ ${ }^{c}$ Yukawa Institute for Theoretical Physics, Kyoto University, \\ Kyoto 606-8502, Japan \\ ${ }^{d}$ Department of Physics, Kyoto University, \\ Kyoto 606-8502, Japan \\ E-mail: kobayashi@particle.sci.hokudai.ac.jp, \\ yujiomur@eken.phys.nagoya-u.ac.jp, takayama@yukawa.kyoto-u.ac.jp, \\ yasuhara@gauge.scphys.kyoto-u.ac.jp
}

ABSTRACT: Flavor symmetric model is one of the attractive Beyond Standard Models (BSMs) to reveal the flavor structure of the Standard Model (SM). A lot of efforts have been put into the model building and we find many kinds of flavor symmetries and setups are able to explain the observed fermion mass matrices. In this paper, we look for common predictions of physical observables among the ones in flavor symmetric models, and try to understand how to test flavor symmetry in experiments. Especially, we focus on the BSMs for leptons with extra Higgs $\mathrm{SU}(2)_{L}$ doublets charged under flavor symmetry. In many flavor models for leptons, remnant symmetry is partially respected after the flavor symmetry breaking, and it controls well the Flavor Changing Neutral Currents (FCNCs) and suggests some crucial predictions against the flavor changing process, although the remnant symmetry is not respected in the full lagrangian. In fact, we see that $\tau^{-} \rightarrow e^{+} \mu^{-} \mu^{-}\left(\mu^{+} e^{-} e^{-}\right)$ and $e^{+} e^{-} \rightarrow \tau^{+} \tau^{-}\left(\mu^{-} \mu^{+}\right)$processes are the most important in the flavor models that the extra Higgs doublets belong to triplet representation of flavor symmetry. For instance, the stringent constraint from the $\mu \rightarrow e \gamma$ process could be evaded according to the partial remnant symmetry. We also investigate the breaking effect of the remnant symmetry mediated by the Higgs scalars, and investigate the constraints from the flavor physics: the flavor violating $\tau$ and $\mu$ decays, the electric dipole moments, and the muon anomalous magnetic moment. We also discuss the correlation between FCNCs and nonzero $\theta_{13}$, and point out the physical observables in the charged lepton sector to test the BSMs for the neutrino mixing.

Keywords: Discrete and Finite Symmetries, Spontaneous Symmetry Breaking

ARXIV EPRINT: 1505.07636 


\section{Contents}

1 Introduction 1

2 Generic argument about FCNCs in flavor models 3

2.1 Remnant symmetry in flavor symmetric models 3

2.2 Setup 4

2.3 Mass base of charged leptons 5

2.4 Mass base of scalars 5

2.5 Yukawa couplings 6

$\begin{array}{lll}3 & \text { Study of the Higgs potential } & 7\end{array}$

4 Flavor physics $\quad 9$

4.1 $\mathcal{T}$-conserving contributions $\quad 9$

4.1.1 $\mathcal{T}$-charged scalar interactions $\quad 9$

$\begin{array}{lll}\text { 4.1.2 } & \mathcal{T} \text {-trivial scalar interactions } & 12\end{array}$

$\begin{array}{lll}4.2 & \mathcal{T} \text {-breaking contributions } & 12\end{array}$

$\begin{array}{lll}\text { 4.2.1 Constraint from the } \mu \rightarrow e \gamma \text { process } & 13\end{array}$

$\begin{array}{lll}\text { 4.2.2 } & \text { Constraint from the } \tau \rightarrow e \gamma \text { process } & 14\end{array}$

4.2.3 Muon anomalous magnetic moment $(g-2)_{\mu} \quad 14$

$\begin{array}{lll}4.3 & \text { Short summary } & 15\end{array}$

5 Mass mixing induced by $\mathcal{T}$-breaking terms $\quad 16$

$\begin{array}{lll}6 & \text { Summary } & 22\end{array}$

A Mass matrices and vacuum alignment in $A_{4}$ model 24

\section{Introduction}

We know that there are three generations of fermions in nature. Each generation carries the same quantum number, and only their masses are different from each other. In the Standard Model (SM), the transition among the generations occurs only through the weak boson exchanging, but the flavor-changing processes via the Flavor Changing Neutral Currents (FCNCs) are strongly suppressed because of the Glashow-Iliopoulos-Maiani (GIM) mechanism [1]. This SM picture successfully describes the experimental results, but we may wonder why such a flavor structure exists in our nature. We expect that Beyond Standard Model (BSM) exists out of our current experimental reach, and reveals the origin of the three generations. One of the promising BSMs is a flavor symmetric model.

In the SM, flavor symmetry is explicitly broken by Yukawa couplings to generate fermion mass matrices. Without the couplings, we could find SU(3) symmetry in each sector 
of left-handed (right-handed) up-type, down-type quarks and leptons. In flavor symmetric models, the $\mathrm{SU}(3)$ symmetry or the subgroup of $\mathrm{SU}(3)$ is respected in the Lagrangian, introducing extra scalar bosons charged under the flavor symmetry. For instance, additional

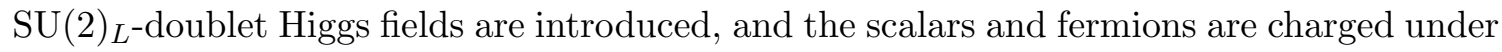
the flavor symmetry to write down Yukawa couplings. The flavor-charged Higgs fields develop nonzero vacuum expectation values (VEVs), and break not only the electro-weak (EW) symmetry but also the flavor symmetry. Then the flavor structure of the SM is effectively generated at the low-energy scale. This BSM would be very attractive and reasonable, and so many types of flavor symmetric models have been proposed so far [2-4]. Especially, we could find so many models motivated by the large mixing in neutrino sector, because the experimental result may imply so-called Tri-Bi maximal mixing [5-7], which can be easily accommodated by the BSMs with non-Abelian discrete flavor symmetry such as $A_{4}$ [8-12], $S_{4}$ [13-15], and $\Delta(27)$ [16] etc.. Recent result on the nonzero $\theta_{13}$ [17-22] may require some small modifications in those models, but we could expect that so many kinds of flavor symmetric models can be still consistent with the experimental results [4, 23-43]. Then, the next question in this approach to the flavor structure would be how to test the flavor symmetry in experiments.

One hint to clarify which kinds of symmetry exist behind the flavor structure would be obtained, if we consider the origin of the remnant symmetry in the fermion mass matrices in the SM. As we discuss in section 2, we see that there is symmetry in mass matrices of leptons in the SM, which is explicitly broken by the weak interaction involving $W$ boson. If flavor symmetry exists behind the SM, the remnant symmetry might be the fragment of the flavor symmetry broken at high energy. In fact, one can find a lot of works on flavor models, based on the assumption that the symmetry in the fermion mass matrices is the subgroup of flavor symmetry [2-4, 26, 44].

In this paper, we investigate especially FCNCs involving charged leptons in flavor symmetric models, where the symmetry in the charged lepton mass matrix is the subgroup of the flavor symmetry and extra $\mathrm{SU}(2)_{L}$-doublet Higgs fields charged under the additional symmetry are introduced. Once we assume that the symmetry is originated from the flavor symmetry spontaneously broken at high energy, we find that the FCNCs involving the extra Higgs fields are predicted by the remnant symmetry. The remnant symmetry would not be respected in the full Lagrangian, but it could well control the FCNCs as long as the breaking terms are enough small in the Higgs potential. In section 2, we discuss the remnant symmetry and our setup in this paper. Then we investigate the FCNCs involving neutral scalars and charged leptons, and discuss Higgs potential in section 3. We see that the partially remnant symmetry in the lepton mass matrix well controls FCNCs, and study our signals and current experimental constraints in flavor physics in section 4 . On the other hand, it is also one of crucial issues to understand how to derive nonzero $\theta_{13}$ in flavor symmetric models. As we mentioned above, nonzero $\theta_{13}$ may require some modifications in conventional setups, because simple scenarios tend to predict vanishing $\theta_{13}$. We study the correlation between $\theta_{13}$, especially given by the mixing angles in charged lepton sector, and FCNCs, in section 5. Section 6 is devoted to summary. In the appendix A, we introduce the $A_{4}$ model as a concrete example. 


\section{Generic argument about FCNCs in flavor models}

In the SM, the fermions obtain masses according to the nonzero VEV of a Higgs field and Yukawa couplings. The Yukawa couplings should be defined to realize the large mass hierarchies and mixing in quarks and lepton sectors, so that the flavor symmetry that rotates the flavors is explicitly broken by the couplings in the SM. Furthermore, the charged currents involving $W$ boson change the flavors, so that it would be difficult to find out even very simple flavor symmetry such as $Z_{2}$ and $Z_{3}$ in the full Lagrangian of the SM.

Now, let us focus on mass matrices of leptons and look for symmetry that is respected in only each mass matrix. For instance, if we see only the mass matrices for the charged lepton $\left(M_{l}\right)$ and the neutrinos $\left(M_{N}\right)$, we could find flavor symmetry as,

$$
M_{l} M_{l}^{\dagger}=T_{L} M_{l} M_{l}^{\dagger} T_{L}^{\dagger}, \quad M_{l}^{\dagger} M_{l}=T_{R} M_{l}^{\dagger} M_{l} T_{R}^{\dagger}, \quad M_{N}=S^{T} M_{N} S,
$$

assuming neutrinos are Majorana particles (See ref. [4], for instance). When $M_{l}$ and $M_{N}$ are diagonal, $T_{L}, T_{R}$, and $S$ could be described as

$$
T_{L}=\left(\begin{array}{ccc}
1 & 0 & 0 \\
0 & \eta_{L} & 0 \\
0 & 0 & \eta_{L}^{*}
\end{array}\right), \quad T_{R}=\left(\begin{array}{ccc}
1 & 0 & 0 \\
0 & \eta_{R} & 0 \\
0 & 0 & \eta_{R}^{*}
\end{array}\right), \quad S=\left(\begin{array}{ccc}
(-1)^{p} & 0 & 0 \\
0 & (-1)^{q} & 0 \\
0 & 0 & (-1)^{p+q}
\end{array}\right),
$$

where $\eta_{L}$ and $\eta_{R}$ are the complex numbers which satisfy $\eta_{L} \eta_{L}^{*}=\eta_{R} \eta_{R}^{*}=1$, and $p, q$ are integer. $T_{L}, T_{R}$, and $S$ are not conserved in the full Lagrangian. In fact, they are broken by the gauge interaction with $W$ boson explicitly. However, they may give a hint for the mystery of the flavor structure in the SM. As discussed in refs. [2-4, 26, 44], we can find the remnant symmetry, $T_{L}, T_{R}$, and $S$ in the flavor models which explain the realistic mass matrices naturally, and the remnant ones could be interpreted as the subgroup of the original flavor symmetry. Below, let us discuss such a kind of flavor models and $\mathrm{SU}(2)_{L^{-}}$ doublet extra Higgs fields charged under the flavor symmetry, and consider the scenario that the simple remnant symmetry appears after the symmetry breaking.

\subsection{Remnant symmetry in flavor symmetric models}

Let us consider flavor symmetric models with extra Higgs doublets. The extra symmetry may be non-Abelian discrete symmetry, and the extra scalars may belong to non-trivial singlet, doublet or triplet. Let us focus on Yukawa couplings of charged lepton sector in flavor symmetric models. In general, the couplings for the fermion masses could be described as

$$
\mathcal{L}=-\bar{L}_{i} M_{l}(\phi)^{i j} E_{R j}+\text { h.c. }
$$

$\phi$ is a scalar charged under the EW symmetry and the flavor symmetry, and we simply assume that $M_{l}$ only depends on $\phi$. Then $M_{l}$ satisfies the following relation according to the flavor symmetry $(\mathcal{G})$ with generators $(g)$;

$$
M_{l}(\phi)=g_{L} M_{l}\left(g_{\phi}^{\dagger} \phi\right) g_{R} .
$$


$g_{L, R, \phi}$ are defined corresponding to the representations of $L_{i}, E_{R i}$, and $\phi$ under $\mathcal{G}$. When $\phi$ develops the nonzero VEV, the EW and flavor symmetry are broken and mass matrix for charged leptons is generated. Let us simply assume that the remnant symmetry of the flavor symmetry $(\mathcal{T})$, whose generator is $T \subset g$, is still hold in the mass matrix as follows:

$$
\begin{aligned}
M_{l}(\langle\phi\rangle) & =T_{L} M_{l}\left(T_{\phi}^{\dagger}\langle\phi\rangle\right) T_{R}^{\dagger}=T_{L} M_{l}(\langle\phi\rangle) T_{R}^{\dagger}, \\
T_{\phi}^{\dagger}\langle\phi\rangle & =T_{\phi}\langle\phi\rangle=\langle\phi\rangle .
\end{aligned}
$$

Let us consider the case that $L_{i}$ is triplet-representation in the diagonal base of $T_{L}$. Then $T_{L}$ for $L_{i}$ would be,

$$
T_{L}=\left(\begin{array}{ccc}
1 & 0 & 0 \\
0 & \eta_{L} & 0 \\
0 & 0 & \eta_{L}^{*}
\end{array}\right) .
$$

If $\eta_{L}$ is not \pm 1 , we find that $L_{i}$ in the diagonal base of $T_{L}$ is identical to the field $\left(l_{i}\right)$ in the mass base according to the relation of eq. (2.1). In this paper, we only focus on the case with $\eta_{L} \neq \pm 1$. Moreover, we especially discuss flavor models with flavor tripletrepresentation Higgs doublet $\left(\phi \equiv H_{a}\right)$, so that the VEV alignment is given by eqs. (2.6) and (2.7) with $T_{L}=T_{\phi}$ :

$$
\left(\left\langle\phi_{1}\right\rangle,\left\langle\phi_{2}\right\rangle,\left\langle\phi_{3}\right\rangle\right) \propto(1,0,0) .
$$

The orthogonal directions would be in the mass base of scalars around the VEV, and they may also respect the remnant symmetry, $\mathcal{T}$. Furthermore, the mass base of $E_{R i}$ is also fixed by $T_{R}$ as we discuss below, so that we can expect that it is possible that the FCNCs involving Higgs fields are qualitatively discussed in this kind of scenario, not mentioning original symmetry $\mathcal{G}$.

\subsection{Setup}

Below, we focus on flavor models with triplet-representation Higgs doublet $H_{a}$. The Yukawa coupling for charged lepton is given by

$$
\mathcal{L}=-\bar{L}_{i} Y_{i a}^{j} H_{a} E_{R}^{j}+\text { h.c. },
$$

where $i, j=e, \mu, \tau$ and $a=1,2,3$. The texture of the matrices, $Y_{i a}^{j}$, is fixed by $\mathcal{G}$, and we can find this type of setups in refs. [4, 8, 9, 27, 45-49]. ${ }^{1}$ Our assumptions of our setup are as follows:

- $L_{i}$ and $H_{a}$ are triplet representations of $\mathcal{G}$,

- $\left\langle H_{a}\right\rangle$ breaks $\mathcal{G}$ to $\mathcal{T}$,

- $E_{R i}$ is non-trivial singlet of $\mathcal{T}$.

$\mathcal{T}$ would not be conserved in the full Lagrangian but partially respected, i.e. in the charged lepton Yukawa couplings. As discussed in subsection 2.1, charged lepton mass matrices only hold the symmetry as in eq. (2.5). It is well-known that this situation successfully realizes

\footnotetext{
${ }^{1} \mathrm{SU}(2)_{L}$ singlets charged under flavor symmetry are introduced allowing higher-dimensional operators in refs. [10, 28-32]. Such kind of models are not be considered in this paper, but could be also related to our studies.
} 
realistic mass matrices according to the Tri-Bi maximal mixing structure in flavor models with non-Abelian discrete symmetry: for instance $A_{4}$ [8-10], $S_{4}$ [4, 13-15, 24, 26, 50], $A_{5}[51,52], T_{7}[45,46], \Delta(27)[27]$, and $\Delta\left(6 n^{2}\right)$ [53-60]. Note that $E_{R i}$ may belong to the triplet-representation or non-trivial singlet of $\mathcal{G}$ before the symmetry breaking, but we do not specify it.

\subsection{Mass base of charged leptons}

$L_{i}$ and $H_{a}$ are triplet-representation of $\mathcal{G}$, so that they are also the triplet of $\mathcal{T}$. Let us denote $L_{i}$ by the fields $\left(l_{i}\right)$ in the diagonal base of $T_{L}$. Then $l_{i}$ are in the mass base as we discussed above. Let us denote $E_{R i}$ as $e_{R i}$ in this base. Then, $e_{R i}$ are also the ones in the mass base, which transform as $\left(e_{R 1}, e_{R 2}, e_{R 3}\right) \rightarrow\left(e_{R 1}, \eta_{L} e_{R 2}, \eta_{L}^{*} e_{R 3}\right)$, because of eq. (2.5). Eventually, the texture of $Y_{i a}^{j}$ is almost fixed because of $T_{L}$ in eq. (2.7):

$$
\left(Y_{i a}^{1}\right)=\frac{\sqrt{2}}{v \cos \beta}\left(\begin{array}{ccc}
m_{1} & 0 & 0 \\
0 & b_{1} & 0 \\
0 & 0 & c_{1}
\end{array}\right),\left(Y_{i a}^{2}\right)=\frac{\sqrt{2}}{v \cos \beta}\left(\begin{array}{ccc}
0 & 0 & c_{2} \\
m_{2} & 0 & 0 \\
0 & b_{2} & 0
\end{array}\right), \quad\left(Y_{i a}^{3}\right)=\frac{\sqrt{2}}{v \cos \beta}\left(\begin{array}{ccc}
0 & b_{3} & 0 \\
0 & 0 & c_{3} \\
m_{3} & 0 & 0
\end{array}\right),
$$

where, $\left\langle H_{1}\right\rangle=v \cos \beta / \sqrt{2}$. See eq. (2.13). Nonzero $b_{2}$ and $c_{3}$ imply $\mathcal{T}=Z_{3}{ }^{2}$ If $Y_{i a}^{j}$ is given by $Y_{i a}^{j}=y^{k} S_{i a}^{k}$, where $S_{i a}^{k}$ is defined by the multiplication rule of $\mathcal{G}$ and $y^{k}$ are dimensionless couplings, the elements of $Y_{i a}^{j}$ could be estimated, substituting

$$
\left|b_{j}\right|=\left|c_{j}\right|=m_{j}
$$

where $m_{j}$ are the charged lepton masses. In this case, the mass matrix for charged lepton $\left(\left(M_{l}\right)_{i}^{k}\right)$ is given by

$$
\left(M_{l}\right)_{i}^{k}=\frac{v \cos \beta}{\sqrt{2}} Y_{i 1}^{k}
$$

Below, we discuss flavor physics assuming the relation in eq. (2.11).

\subsection{Mass base of scalars}

After the EW and flavor symmetry breaking, we find several scalars: CP-even, CP-odd, and charged scalars. In addition to flavor-triplet $H_{a}$, we introduce one flavor-singlet Higgs field, $H_{q}$, in order to realize the realistic mass matrices for quarks. We may need another flavor-charged scalars, $\Phi$, to generate Majorana mass matrices for neutrino mixing and masses. $\Phi$ may break the subgroup $\mathcal{T}$, and the mixing between $H_{a}$ and $\Phi$ may be allowed in the lagrangian. The mixing term may break the vacuum alignment as discussed in eq. (2.6), because the VEV of $\Phi$ corresponds to the $\mathcal{T}$ breaking term. Below, we give some discussion about the mixing, and let us study $H_{a}$ and $H_{q}$, first.

Let us decompose the scalars as follows:

$$
H_{q}=\left(\begin{array}{c}
H_{q}^{+} \\
\frac{1}{\sqrt{2}}\left(v \sin \beta+H_{q}^{0}+i \chi_{q}\right)
\end{array}\right), \quad H_{1}=\left(\begin{array}{c}
H_{1}^{+} \\
\frac{1}{\sqrt{2}}\left(v \cos \beta+H_{1}^{0}+i \chi_{1}\right)
\end{array}\right)
$$

\footnotetext{
${ }^{2}$ In our analysis, we assume the relation of eq. (2.11), so $\mathcal{T}$ is set to $Z_{3}$.
} 
and

$$
H_{2}=\left(\begin{array}{c}
H_{2}^{+} \\
\phi_{2}
\end{array}\right), \quad H_{3}=\left(\begin{array}{c}
H_{3}^{+} \\
\phi_{3}
\end{array}\right) .
$$

$H_{q}$ and $H_{1}$ generally mix each other because they develop nonzero VEVs:

$$
\begin{aligned}
\left(\begin{array}{l}
H_{q}^{+} \\
H_{1}^{+}
\end{array}\right) & =\left(\begin{array}{c}
\cos \beta \\
\sin \beta
\end{array}\right) G^{+}+\left(\begin{array}{c}
-\sin \beta \\
\cos \beta
\end{array}\right) H_{S}^{+}, \\
\left(\begin{array}{l}
\chi_{q} \\
\chi_{1}
\end{array}\right) & =\left(\begin{array}{c}
\cos \beta \\
\sin \beta
\end{array}\right) G^{0}+\left(\begin{array}{c}
-\sin \beta \\
\cos \beta
\end{array}\right) A_{S}, \\
\left(\begin{array}{l}
H_{q}^{0} \\
H_{1}^{0}
\end{array}\right) & =\left(\begin{array}{c}
\cos \alpha \\
\sin \alpha
\end{array}\right) H_{S 1}^{0}+\left(\begin{array}{c}
-\sin \alpha \\
\cos \alpha
\end{array}\right) H_{S 2}^{0} .
\end{aligned}
$$

$G^{0}$ and $G^{+}$are the Goldstone boson eaten by $Z$ and $W^{+}$bosons. If $\mathcal{T}$ is conserved in the mass matrices of scalars, $H_{S}^{+}, A_{S}^{+}, H_{S 1}^{0}$, and $H_{S 2}^{0}$ are in the mass bases, and they do not mix with $H_{2}$ and $H_{3}$ for the $\mathcal{T}$ charge conservation. We will give some discussions about the mixing in section 3 .

$\alpha$ is the mixing angle between two CP-even scalars, and fixed by Higgs potential. If we build Higgs potential to lead SM-like Higgs mass and signal strength, $\alpha$ should be identical to $\beta$, and $H_{S 1}^{0}$ is interrupted as the SM Higgs.

On the other hand, $H_{2}^{+}, H_{3}^{+}, \phi_{2}$ and $\phi_{3}$ are the complex scalars which carry the $\mathcal{T}$ charges: $H_{2} \rightarrow \eta H_{2}$ and $H_{3} \rightarrow \eta^{*} H_{3}$. In general, $H_{2}$ and $H_{3}$ would mix each other according to the nonzero $\operatorname{VEV}\langle\Phi\rangle$, because $\langle\Phi\rangle$ breaks $\mathcal{T}$ spontaneously. We discuss the effect against the observables in flavor physics later.

\subsection{Yukawa couplings}

Now we define $\mathcal{T}$-conserving Yukawa couplings involving scalars. Based on the above argument, we find the following Yukawa couplings which induce flavor violations:

$$
\left.\mathcal{L}_{\mathcal{T}}=-Y_{i 2}^{j} \phi_{2} \bar{l}_{i} e_{R j}-Y_{i 3}^{j} \phi_{3} \bar{l}_{i} e_{R j}-\left(V^{\dagger}\right)_{i k} Y_{k 2}^{j} H_{2}^{+} \bar{\nu}_{L_{i}} e_{R j}-\left(V^{\dagger}\right)_{i k} Y_{k 3}^{j} H_{3}^{+} \bar{\nu}_{L_{i}} e_{R j}+h . \notin 2.18\right)
$$

where $V$ is the PMNS matrix. $Y_{i 2}^{j}$ and $Y_{i 3}^{j}$ are,

$$
\left(Y_{i 2}^{j}\right)=\frac{\sqrt{2}}{v \cos \beta}\left(\begin{array}{ccc}
0 & 0 & b_{3} \\
b_{1} & 0 & 0 \\
0 & b_{2} & 0
\end{array}\right),\left(Y_{i 3}^{j}\right)=\frac{\sqrt{2}}{v \cos \beta}\left(\begin{array}{ccc}
0 & c_{2} & 0 \\
0 & 0 & c_{3} \\
c_{1} & 0 & 0
\end{array}\right) .
$$

As we mentioned above, the complex scalars may not be in the mass bases, because of $\mathcal{T}$ breaking effects in the Higgs potential. In section 4, we investigate the FCNC contributions to flavor physics in the $\mathcal{T}$-conserving limit, and then discuss the corrections from the $\mathcal{T}$ breaking terms in the Higgs potential to the observables in flavor physics. In fact, the breaking effect is strongly constrained by the $\mu \rightarrow e \gamma$ process. 
On the other hand, the neutral and charged scalars from $H_{q}$ and $H_{1}$ consist of Yukawa couplings that are the same as the model called type-X 2HDM in [61], or lepton-specific $2 \mathrm{HDM}$ in [62]:

$$
\begin{aligned}
\mathcal{L}_{2 \mathrm{HDM}}= & -\frac{m_{i} \sin \alpha}{v \cos \beta} H_{S}^{0} \bar{l}_{i} e_{R i}-\frac{m_{i} \cos \alpha}{v \cos \beta} H_{S}^{0} \bar{l}_{i} e_{R i}+\text { h.c. } \\
& -i \frac{m_{i}}{v \tan \beta} A_{S} \bar{l}_{i} e_{R i}-V_{i j} \frac{m_{j}}{v \tan \beta} H_{S}^{+} \bar{\nu}_{L i} e_{R j}+\text { h.c.. }
\end{aligned}
$$

The phenomenology of lepton-specific $2 \mathrm{HDMs}$ has been studied well in refs. [61, 62].

\section{Study of the Higgs potential}

Before studying the phenomenological aspects, let us discuss Higgs potential in flavor symmetric models. In our setup, flavor-triplet $H_{a}$ develops nonzero VEV in the direction of $\left(\left\langle H_{1}\right\rangle,\left\langle H_{2}\right\rangle,\left\langle H_{3}\right\rangle\right) \propto(1,0,0)$, and $\mathcal{T}$ is not broken. $\Phi_{i}$ is $\mathrm{SU}(2)_{L}$-singlet and breaks $\mathcal{G}$ to the subgroup $\mathcal{S}$ of $\mathcal{G}$. In general, $\mathcal{S}$ and $\mathcal{T}$ are not commutative, so that $\left\langle\Phi_{a}\right\rangle$ breaks $\mathcal{T}$ and how to realize the vacuum alignment may be one of the issues in our models. For instance, the mechanism to achieve rigid vacuum alignment has been proposed so far [63].

In order to realize the vacuum alignment that respects $\mathcal{T}$, especially mixing term between $H_{a}$ and $\Phi_{a}$ should be controlled. In general, the Higgs potential is written as

$$
V=V_{H}\left(H_{q}, H_{a}\right)+V_{\Phi}\left(\Phi_{a}, H_{q}\right)+\Delta V\left(\Phi_{a}, H_{a}, H_{q}\right),
$$

where $\Delta V$ only has the mixing terms between $\Phi_{a}$ and $H_{a}$ such as $\left|H_{a}\right|^{2}\left|\Phi_{b}\right|^{2}$ and $H_{a}^{\dagger} H_{q} \Phi_{b}$. If $\Delta V$ is absent, the vacuum alignment of $\left\langle H_{a}\right\rangle$ and $\left\langle\Phi_{a}\right\rangle$ are independently fixed by $V_{H}$ and $V_{\Phi}$. In this case, the mass matrices for the scalars originated from $H_{a}$ and $H_{q}$ would respect $\mathcal{T}$-symmetry, while the mass matrices from $H_{q}$ and $\Phi_{a}$ would respect $\mathcal{S}$-symmetry. This means that scalar mass eigenstates are decided according to only the remnant symmetries in each sector, and the flavor violating Yukawa couplings in the mass base of scalars are given by eq. (2.19).

We consider an example to illustrate our argument. In the absence of $\Delta V$, we can write down the mass matrix for the $\mathrm{CP}$-even scalar mass eigenstates after the spontaneous symmetry breaking. On the basis of $\left(H_{q}^{0}, H_{1}^{0}, H_{2}^{0}, H_{3}^{0}, \Phi_{1}^{0}, \Phi_{2}^{0}, \Phi_{3}^{0}\right)^{T}$, where $\phi_{2}=\frac{1}{\sqrt{2}}\left(H_{2}^{0}+\right.$ $\left.i A_{2}\right), \phi_{3}=\frac{1}{\sqrt{2}}\left(H_{3}^{0}+i A_{3}\right)$ and $\Phi_{j}=\frac{1}{\sqrt{2}}\left(v_{\Phi}+\Phi_{j}^{0}+i A_{j}^{\Phi}\right)$ are defined, the mass matrix of the $\mathrm{CP}$-even mass scalars is given in the following form:

$$
\left(\begin{array}{c|cc}
M_{q}^{2} & m_{H}^{2 T} & m_{\Phi}^{2 T} \\
\hline m_{H}^{2} & M_{H}^{2} & 0 \\
m_{\Phi}^{2} & 0 & M_{\Phi}^{2}
\end{array}\right) .
$$

Here, $m_{H}^{2}$ and $m_{\Phi}^{2}$ are 3 -vectors, $M_{H}^{2}$ and $M_{\Phi}^{2}$ are $3 \times 3$ matrices. $M_{q}^{2}$ is the mass term for $H_{q}^{0}$. The form of submatrices $M_{H}^{2}, M_{\Phi}^{2}$ and sub-vectors $m_{H}^{2}, m_{\Phi}^{2}$ are fixed by the $\mathcal{T}$-conserving and $\mathcal{S}$-conserving conditions,

$$
\begin{aligned}
T_{i k}^{H} M_{H k l}^{2} T_{l j}^{H \dagger} & =M_{H i j}^{2}, & S_{i k}^{\Phi} M_{\Phi k l}^{2} S_{l j}^{\Phi \dagger} & =M_{\Phi i j}^{2}, \\
T_{i k}^{H} m_{H k}^{2} & =m_{H i}^{2}, & S_{i k}^{\Phi} m_{\Phi k}^{2} & =m_{\Phi i}^{2},
\end{aligned}
$$


where $T^{H}$ and $S^{\Phi}$ are generators of subgroups $\mathcal{T}$ and $\mathcal{S}$ in the triplet representation. Similarly, mass matrices for CP-odd and charged scalar mass eigenstates are also determined only by the remnant flavor symmetry in each sector. We show the most simple example with $\mathcal{G}=A_{4}, \mathcal{T}=Z_{3}$, and $\mathcal{S}=Z_{2}$ in appendix A.

Especially, on the base in which $\mathcal{T}$-generator is diagonal, subgroup $\mathcal{T}$ restricts the mass terms for scalar bosons $\left(H_{q}^{0}, H_{1}^{0}, H_{2}^{0}, H_{3}^{0}\right)$ which interact with the SM particles as follows:

$$
\left(\begin{array}{cccc}
M_{q}^{2} & m_{H 1}^{2} & 0 & 0 \\
m_{H 1}^{2} & M_{H 11}^{2} & 0 & 0 \\
0 & 0 & M_{H 22}^{2} & 0 \\
0 & 0 & 0 & M_{H 33}^{2}
\end{array}\right) .
$$

$\mathcal{T}$ completely determines mass matrix for $H_{q}, H_{a}$ because $\mathcal{G}$-singlet boson $H_{q}$ also respects residual symmetry $\mathcal{T}$. Although $H_{q}^{0}, H_{1}^{0}$ and $\Phi_{a}^{0}$ may mix each other because of nonzero $m_{H 1}^{2}$ and $m_{\Phi i}^{2}$, the mass matrix of the scalar bosons can be described in the model independent way as far as $\Delta V=0$.

Scalar mass eigenstates in the case of $\Delta \boldsymbol{V} \neq 0$. Let us consider the case with nonzero $\Delta V$. We simply assume that the nonzero VEV of $\Phi_{a}$ is higher than the EW scale, and we could write down the $\mathcal{T}$-conserving and $\mathcal{T}$-breaking effective potential for $H^{a}$ and $H^{q}$ at the renormalizable level:

$$
\begin{aligned}
& V_{\text {eff }}=V_{\mathcal{T} c}+V_{\mathcal{T} b}, \\
& V_{\mathcal{T} c}=m_{q}^{2}\left|H_{q}\right|^{2}+\left(m_{q 1}^{2} H_{q}^{\dagger} H_{1}+h . c .\right)+m_{1}^{2}\left|H_{1}\right|^{2}+m_{2}^{2}\left|H_{2}\right|^{2}+m_{3}^{2}\left|H_{3}\right|^{2}+V_{T}^{(4)}, \\
& V_{\mathcal{T} b}=m_{q 2}^{2} H_{q}^{\dagger} H_{2}+m_{q 3}^{2} H_{q}^{\dagger} H_{3}+m_{12}^{2} H_{1}^{\dagger} H_{2}+m_{13}^{2} H_{1}^{\dagger} H_{3}+m_{23}^{2} H_{2}^{\dagger} H_{3}+\text { h.c. },
\end{aligned}
$$

where $V_{T}^{(4)}$ is the function which only include $\mathcal{T}$-conserving quartic couplings of $H_{q}$ and $H_{i} . V_{\mathcal{T} c}$ and $V_{\mathcal{T} b}$ are the $\mathcal{T}$-conserving and $\mathcal{T}$-breaking potentials. The scalars from $\Phi$ are omitted assuming that they decouple below the EW scale because of the hierarchy between the VEV of $\Phi$ and the VEVs of Higgs doublets. $V_{\mathcal{T} b}$ is generated by $\Delta V$ in eq. (3.1). We could write quartic couplings in $V_{\mathcal{T} b}$, but the dimensionless couplings are expected to be small, because they are generated by the high-dimensional operators in $V$ or integrating out the heavy scalars in $\Phi .{ }^{3}$ Now, let us consider the stationary conditions for $H_{2}$ and $H_{3}$. In our setup, they should not develop nonzero VEVs to respect $\mathcal{T}$. The defivatives $\partial_{H_{I}} V$ $(I=2,3)$ under the $\mathcal{T}$-conserving conditions depend on the $\mathcal{T}$-breaking terms as,

$$
\partial_{H_{I}} V_{\mathrm{eff}}=m_{q I}^{2} H_{q}^{\dagger}+m_{1 I}^{2} H_{1}^{\dagger}
$$

This leads the equation to realize the vacuum alignment where $\left\langle H_{I}\right\rangle=0$ is satisfied:

$$
m_{q I}^{2} \sin \beta+m_{1 I}^{2} \cos \beta=0 .
$$

In general, the stationary conditions for $H_{1}$ and $H_{q}$ are independent of this condition, so that the fine-tuning would be required, if $m_{q I}^{2}=m_{1 I}^{2}=0$ is not satisfied. The quartic

\footnotetext{
${ }^{3}$ Our argument in this section would be also reasonable, as far as $\mathcal{T}$-breaking quartic couplings are tiny.
} 
couplings in $V_{\text {eff }}$ respect the remnant symmetry $\mathcal{T}$, so that $m_{q I}^{2}$ and $m_{1 I}^{2}$ only contribute to the mass mixing between $\mathcal{T}$-charged scalars and $\mathcal{T}$-trivial scalars. We could conclude that $\mathcal{T}$-charged scalars do not mix with $\mathcal{T}$-trivial scalars, unless we admit the tuning in eq. (3.8).

On the other hand, the mass mixing between $H_{2}$ and $H_{3}$ generated by $m_{23}^{2}$ would not be controlled by the vacuum alignment, because the both VEVs of $H_{2}$ and $H_{3}$ vanish. We discuss the $\mathcal{T}$-breaking effects in section 4.2. We also study the $\mathcal{T}$-breaking terms corresponding to mixing between the $\mathcal{T}$-trivial and the $\mathcal{T}$-charged scalars, in section 5 .

Note that the scalars in $\Phi_{a}$ may have the low mass compatible with the EW scale, and they may mix with $H_{2}$ and $H_{3}$, although the mixing given by $\Delta V$ should be controlled to realize the vacuum alignment. Moreover, $\Phi_{a}$ dominantly couple with neutrinos, even if the mixing exists. Eventually we discuss phenomenology in the limit that the mixing with the scalars in $\Phi_{a}$ is negligible.

\section{Flavor physics}

We have seen that the FCNCs involving scalars are well controlled, if we assume that the partially remnant symmetry $\mathcal{T}$ is respected in the Yukawa couplings. Even if $\mathcal{T}$ is broken in the Higgs potential, we could expect that it is possible to discuss the contributions of the FCNCs to flavor physics as far as the breaking effect is smaller than the $\mathcal{T}$-conserving one. As discussed in subsection 2.5, the $\mathcal{T}$-conserving FCNCs are distinguishing, so that we could qualitatively analyze their signals in flavor physics. In the section 4.1 , we consider the $\mathcal{T}$-conserving case and then we will see the $\mathcal{T}$-breaking effect including the loop corrections in subsection 4.2 .

\section{1 $\mathcal{T}$-conserving contributions}

First of all, let us discuss the flavor physics in the case that $\mathcal{T}$ is conserved in charged leptons and scalar mass matrices. The Yukawa couplings between scalars and charged leptons are given by eqs. (2.19) and (2.20). $\phi_{2,3}$ and $H_{2,3}^{+}$are the mass eigenstates in this case. The $\mathcal{T}$-charged scalar masses are also expected to be around the EW scale, because the masses are given by $V_{H}$, so we are especially interested in the EW-scale masses of the scalars.

\subsection{1 $\mathcal{T}$-charged scalar interactions}

Through the exchanging of $\phi_{2,3}$, (flavor-changing) 4 -fermi interactions are effectively generated as

$$
\begin{aligned}
\mathcal{L}_{\mathcal{T}}^{(4)}= & \frac{1}{v^{2} \cos ^{2} \beta}\left\{\frac{\left|b_{3}\right|^{2}}{m_{\phi_{2}}^{2}}\left(\overline{\tau_{R}} e_{L}\right)\left(\overline{e_{L}} \tau_{R}\right)+\frac{\left|b_{2}\right|^{2}}{m_{\phi_{2}}^{2}}\left(\overline{\mu_{R}} \tau_{L}\right)\left(\overline{\tau_{L}} \mu_{R}\right)+\frac{\left|b_{1}\right|^{2}}{m_{\phi_{2}}^{2}}\left(\overline{e_{R}} \mu_{L}\right)\left(\overline{\mu_{L}} e_{R}\right)\right. \\
& +\frac{\left|c_{3}\right|^{2}}{m_{\phi_{3}}^{2}}\left(\overline{\tau_{R}} \mu_{L}\right)\left(\overline{\mu_{L}} \tau_{R}\right)+\frac{\left|c_{2}\right|^{2}}{m_{\phi_{3}}^{2}}\left(\overline{\mu_{R}} e_{L}\right)\left(\overline{e_{L}} \mu_{R}\right)+\frac{\left|c_{1}\right|^{2}}{m_{\phi_{3}}^{2}}\left(\overline{e_{R}} \tau_{L}\right)\left(\overline{\tau_{L}} e_{R}\right) \\
& +\frac{b_{2}^{*} b_{3}}{m_{\phi_{2}}^{2}}\left(\overline{\mu_{R}} \tau_{L}\right)\left(\overline{e_{L}} \tau_{R}\right)+\frac{b_{1}^{*} b_{2}}{m_{\phi_{2}}^{2}}\left(\overline{e_{R}} \mu_{L}\right)\left(\overline{\tau_{L}} \mu_{R}\right)+\frac{b_{3}^{*} b_{1}}{m_{\phi_{2}}^{2}}\left(\overline{\tau_{R}} e_{L}\right)\left(\overline{\mu_{L}} e_{R}\right)+\text { h.c. } \\
& \left.+\frac{c_{2}^{*} c_{3}}{m_{\phi_{3}}^{2}}\left(\overline{\mu_{R}} e_{L}\right)\left(\overline{\mu_{L}} \tau_{R}\right)+\frac{c_{1}^{*} c_{2}^{2}}{m_{\phi_{3}}^{2}}\left(\overline{e_{R}} \tau_{L}\right)\left(\overline{e_{L}} \mu_{R}\right)+\frac{c_{3}^{*} c_{1}^{2}}{m_{\phi_{3}}^{2}}\left(\overline{\tau_{R}} \mu_{L}\right)\left(\overline{\tau_{L}} e_{R}\right)+\text { h.c. }\right\} .
\end{aligned}
$$



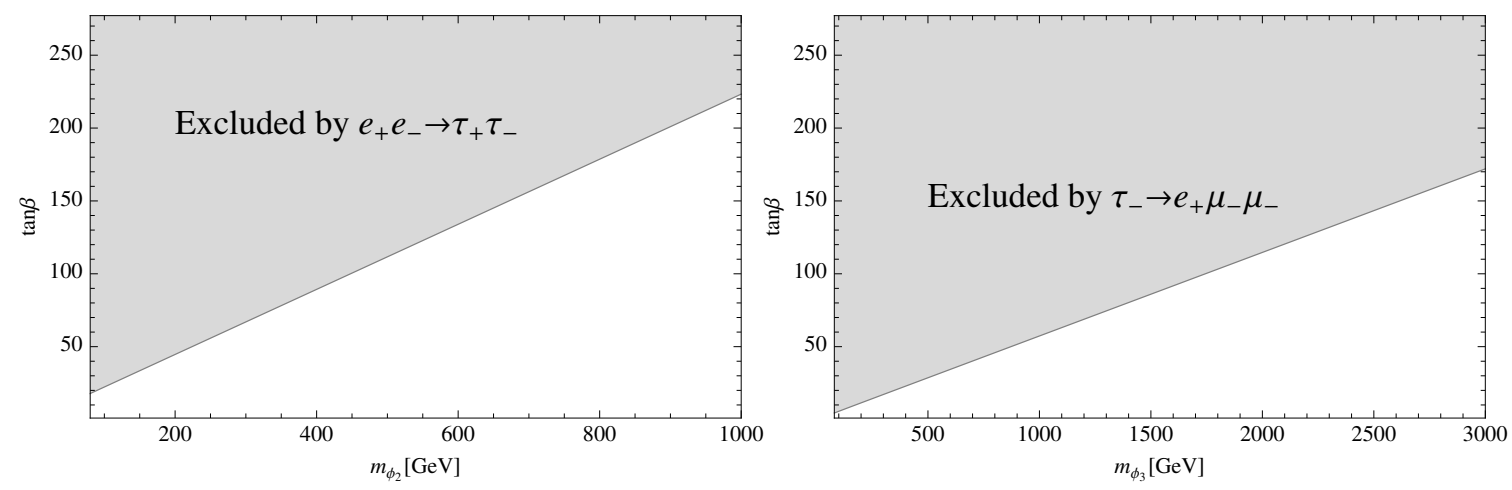

Figure 1. $m_{\phi_{2,3}}$ and $\tan \beta$. The gray region is excluded by $e^{+} e^{-} \rightarrow \tau^{+} \tau^{-}$at the LEP experiment and the LFV $\tau$ decay, $\tau^{-} \rightarrow e^{+} \mu^{-} \mu^{-}$.

The charged Higgs scalars $H_{2,3}^{+}$also induce flavor violation, and it is derived by replacing $\overline{l_{L}^{i}}$ with $\overline{\nu_{L}^{k}} V_{k i}$. The mass difference between $H_{2,3}^{+}$and $\phi_{2,3}$ is strongly constrained by the $\rho$ parameter, so that we set $m_{\phi_{2}}^{2}=m_{H_{2}^{+}}^{2}$ and $m_{\phi_{3}}^{2}=m_{H_{3}^{+}}^{2}$ in our study. The lower bound on the charged Higgs mass is given by the direct search at the LEP experiment: $m_{H^{ \pm}} \gtrsim 80 \mathrm{GeV}$ [64]. Below, we survey the parameter space above the lower mass.

One of the stringent constraints on the flavor violating couplings is from $e^{+} e^{-} \rightarrow l^{+} l^{-}$ $(l=e, \mu, \tau)$ at the LEP [65]. Assuming the relation in eq. (2.11) with $\left(m_{1}, m_{2}, m_{3}\right)=$ $\left(m_{e}, m_{\mu}, m_{\tau}\right)$, the processes $e^{+} e^{-} \rightarrow \mu^{+} \mu^{-}, \tau^{+} \tau^{-}$are enhanced through $\phi_{e \mu}$ exchanging in $t$-channel:

$$
m_{\phi_{2}} \gtrsim 0.62 \times \frac{m_{\tau}}{v \cos \beta} \mathrm{TeV}, m_{\phi_{3}} \gtrsim 2.23 \times \frac{m_{\mu}}{v \cos \beta} \mathrm{TeV}
$$

The allowed region is summarized in figure 1 . If we consider the model which does not hold the relation in eq. (2.11), $m_{\phi_{2}}$ may face the stronger bound from $e^{+} e^{-} \rightarrow \mu^{+} \mu^{-}$, but the bound from the flavor violating $\tau$ decay is stronger than the LEP bound, as we discuss below.

Flavor violating decays of $\tau$ and $\mu$ have been well investigated in the experiments, and the constraints are summarized in refs. [66, 67]. In our models, $\mathcal{T}$-charge should be conserved, so that the final states from $\tau$ and $\mu$ decays should be $\mathcal{T}$-charged states. That is, the possible decay patterns of $\tau$ are only

$$
\tau^{-} \rightarrow \mu^{+} e^{-} e^{-}, e^{+} \mu^{-} \mu^{-}
$$

$\mu \rightarrow 3 e$, which is strongly constrained by the experiments [67], can be forbidden. Assuming the relation in eq. (2.11), the strong bound on the flavor violating decays is obtained from mainly $\tau^{-} \rightarrow e^{+} \mu^{-} \mu^{-}$as $\operatorname{Br}\left(\tau^{-} \rightarrow e^{+} \mu^{-} \mu^{-}\right)<1.7 \times 10^{-8}$ [66] with

$$
\operatorname{Br}\left(\tau^{-} \rightarrow e^{+} \mu^{-} \mu^{-}\right)=\frac{m_{\tau}^{5}}{3(8 \pi)^{3} \Gamma_{\tau}}\left|\frac{m_{\tau} m_{\mu}}{m_{\phi_{3}}^{2}(v \cos \beta)^{2}}\right|^{2},
$$


where, $\Gamma_{\tau}$ is total $\tau$ decay width. The allowed region is summarized in figure $1 .{ }^{4}$

In addition, the charged Higgs exchanging processes may also contribute to the $\tau$ and $\mu$ decay. The chirality of the charged lepton in the decays is different from the one in the SM, so that the correction may be quite small. Assuming the charged lepton in the final state is massless, the deviation of the leptonic decay is evaluated as,

$$
\Delta \operatorname{Br}\left(l_{i} \rightarrow l_{j} \overline{\nu_{k}} \nu_{n}\right) \simeq \frac{1}{32 G_{F}^{2}} \sum_{a=2,3} \frac{\left|Y_{n a}^{i} Y_{k a}^{* j}\right|^{2}}{m_{H_{a}^{+}}^{4}}
$$

Assuming the relation in the eq. (2.11) and $m_{H_{a}^{+}}=m_{\phi_{a}}$ for the EWPOs, we find that the modified branching ratio of muonic $\tau$ decay is at most,

$$
\operatorname{Br}(\tau \rightarrow \mu \bar{\nu} \nu) \lesssim 1.00107 \times \operatorname{Br}(\tau \rightarrow \mu \bar{\nu} \nu)_{\mathrm{SM}},
$$

including the contribution of $\mu$ mass. $\operatorname{Br}(\tau \rightarrow \mu \bar{\nu} \nu)_{\mathrm{SM}}$ is the SM prediction and this modified value is within the error of the current experimental measurement of the $\tau$ decay [69]. The contribution of charged Higgs carrying also $\mathcal{T}$-charges to the other decay such as $\mu \rightarrow e \nu \nu$ is strongly suppressed by the Yukawa couplings.

Including one-loop corrections involving the extra scalars, the $Z$ and $W$ couplings would be slightly deviated from the SM ones:

$$
\mathcal{L}_{\mathrm{EW}}=g_{Z} Z^{\rho}\left\{\left(q_{L}+\Delta q_{L}^{i}\right) \overline{l_{L}^{i}} \gamma_{\rho} l_{L}^{i}+\left(q_{R}+\Delta q_{R}^{i}\right) \overline{l_{R}^{i}} \gamma_{\rho} l_{R}^{i}+\left(q_{L}^{\nu}+\Delta q_{L}^{\nu i j}\right) \overline{\nu_{L}^{i}} \gamma_{\rho} \nu_{L}^{j}\right\},
$$

where $\left(q_{L}, q_{R}, q_{L}^{\nu}\right)=\left(-1 / 2+\sin ^{2} \theta_{W}, \sin ^{2} \theta_{W}, 1 / 2\right)$ are defined. $\sin \theta_{W}$ is the Weinberg angle, and $g_{Z}$ is the gauge coupling for $Z$-boson interaction. According to the one-loop diagrams involving $\phi_{23}$, they are estimated as follows:

$$
\begin{aligned}
\Delta q_{L}^{i} & =\sum_{a=2,3} \sum_{k=1}^{3} \frac{\left|Y_{i a}^{k}\right|^{2}}{16 \pi^{2}} \frac{M_{Z}^{2}}{m_{\phi_{a}}^{2}}\left(-\frac{1}{36}-\frac{1}{3} \sin ^{2} \theta_{W}\right), \\
\Delta q_{R}^{i} & =\sum_{a=2,3} \sum_{k=1}^{3} \frac{\left|Y_{k a}^{i}\right|^{2}}{16 \pi^{2}} \frac{M_{Z}^{2}}{m_{\phi_{a}}^{2}}\left(\frac{7}{36}-\frac{1}{3} \sin ^{2} \theta_{W}\right), \\
\Delta q_{L}^{\nu i j} & =\sum_{a=2,3} \sum_{k, l, n=1}^{3} V^{* k i} V^{n j} \frac{Y_{k a}^{l} Y_{n a}^{l}}{16 \pi^{2}} \frac{M_{Z}^{2}}{m_{\phi_{a}}^{2}}\left(\frac{1}{36}-\frac{7}{18} \sin ^{2} \theta_{W}\right),
\end{aligned}
$$

where $m_{i} \ll M_{Z}^{2} \ll m_{\phi_{a}}$ is assumed. In the model which satisfies the relation in eq. (2.11), $\Delta q_{L}^{e, \mu}, \Delta q_{R}^{\tau}$, and $\Delta q_{L}^{\nu i j}$ might be sizable. The constraints from $e^{+} e^{-} \rightarrow \tau^{+} \tau^{-}$and $\tau^{-} \rightarrow$ $e^{+} \mu^{-} \mu^{-}$give the maximal sizes of the deviations:

$$
\begin{aligned}
\Delta q_{L}^{e} & \approx 2.87 \times 10^{-5}, \quad \Delta q_{L}^{\mu} \approx-1.89 \times 10^{-6}, \quad \Delta q_{R}^{\tau} \approx 3.21 \times 10^{-5}, \\
\Delta q_{L}^{\nu i j} & \approx-1.7 \times 10^{-5} \times V^{i 3} V^{* j 3} .
\end{aligned}
$$

They are too tiny to compare with the current experimental results. In fact, the maximal values are within the error of the measurements of $Z$ boson [70].

\footnotetext{
${ }^{4}$ The lepton flavor violating $(\mathrm{LFV}) \tau$ decays induced by tree-level FCNCs involving extra scalars have been investigated in a generic two-Higgs-doublet model [68].
} 


\subsection{2 $\mathcal{T}$-trivial scalar interactions}

$H_{q}$ and $H_{1}$ are not charged under $\mathcal{T}$, and they develop nonzero VEVs. Their Yukawa couplings with SM fermions are flavor-diagonal in the mass base, under the $\mathcal{T}$-conserving assumption. Then, we could conclude that $H_{q}$ and $H_{1}$ induce so-call minimal flavor violation, and evade the stringent constraints from flavor physics. This type of scalars with the interactions in eq. (2.20) have been well investigated so far, motivated by, for instance, the deviation of muon anomalous magnetic moment [71, 72].

The lower bound on the charged Higgs mass comes from the direct search for charged Higgs at the LHC and it is given by top mass to evade the exotic top decay: $m_{H^{ \pm}} \gtrsim$ $172 \mathrm{GeV}[73,74]$. The pseudo scalar mass should be close to the charged Higgs mass to avoid too large deviation of the $\rho$ parameter. Flavor changing processes in $B$ physics may constrain our $\mathcal{T}$-trivial scalars. For instance, $B \rightarrow X_{s} \gamma$ process gives the lower bound on $\tan \beta: \tan \beta \gtrsim 1[75] . \operatorname{Br}\left(B^{-} \rightarrow \tau^{-} \nu\right)$ is also slightly deviated from the SM prediction according to the charged Higgs exchanging, but it is less than $1 \%$ in the region with $m_{H^{ \pm}} \geq 172 \mathrm{GeV}$. As pointed out in ref. [71, 72], the discrepancy of muon anomalous magnetic moment may be explained if $\tan \beta$ is quite large and pseudo scalar is rather small. Let us also survey the parameter region in the next section.

\section{$4.2 \quad \mathcal{T}$-breaking contributions}

Next, we investigate the contributions of $\mathcal{T}$-breaking terms to flavor physics. If we can assume that the remnant symmetry $\mathcal{T}$ is respected in Higgs potential after the symmetry breaking, we could expect that only $\mathcal{T}$-symmetric terms are relevant to flavor violating processes. However, as we have seen in section $3, \Delta V$ may be allowed even if we assume that the vacuum alignment respects $\mathcal{T}$ and $\mathcal{S}$. After the symmetry breaking, $\Delta V$ induces $\mathcal{T}$-breaking terms, such as $m_{23}^{2}$ in eq. (3.6), in scalar mass matrices because of nonzero $\langle\Phi\rangle$, so that we may have to control $\Delta V$ to evade the stringent constraints from flavor physics.

$\mathcal{T}$-breaking terms would appear in scalar mass matrices, according to $\Delta V$ in eq. (3.1),

$$
\mathcal{L}_{\mathcal{T}}=-\frac{1}{2}\left(\delta m_{H}^{2}\right)_{a b} H_{a}^{0} H_{b}^{0}-\frac{1}{2}\left(\delta m_{A}^{2}\right)_{a b} A_{a} A_{b}-\left(\delta m_{H^{+}}^{2}\right)_{a b} H_{a}^{+} H_{b}^{-} .
$$

$A_{a}$ and $H_{a}^{+}$denote the two kinds of scalars: $\left\{A_{a}\right\}=\left\{A_{2}, A_{3}\right\}$ and $\left\{H_{a}^{ \pm}\right\}=\left\{H_{2}^{ \pm}, H_{3}^{ \pm}\right\}$. There are two CP-even scalars and they mix each other in general according to $\left(\delta m_{H}^{2}\right)_{a b}$, where $\left\{H_{a}^{0}\right\}=\left\{H_{2}^{0}, H_{3}^{0}\right\}$ is defined.

Besides, there may be mixings between $\mathcal{T}$-trivial and $\mathcal{T}$-charged scalars, such as $\left(\delta m_{H}^{2}\right)_{a 1} H_{a}^{0} H_{S 1}^{0}$, although they require the fine-tuning against the parameters in Higgs potential, as discussed in eq. (3.8). The mixings relate to the vacuum alignment, so we analyze the $\mathcal{T}$-breaking terms including the study about the deviation of the vacuum alignment, in section 5 .

In general, $\Phi$ also predicts extra scalars, and should be involved in the scalar mass matrices. However, $\Phi$ mainly couples with neutrinos, so the constraint on $\Phi^{i}$ is rather weak. Simply we assume that the scalars from $\Phi^{i}$ gain heavy masses according to nonzero VEVs of $\Phi^{i}$ in $V_{\Phi}$, and decouple around the EW scale. 
We could expect that this assumption leads the approximate $\mathcal{T}$-conserving situation with

$$
\left(\delta m_{H}^{2}\right)_{a b},\left(\delta m_{A}^{2}\right)_{a b},\left(\delta m_{H^{+}}^{2}\right)_{a b} \ll m_{\phi_{2,3}}^{2},
$$

and discuss the bound on the breaking effects from the experiments. The relevant constraints are from $l \rightarrow l^{\prime} \gamma$ processes [76, 77]. Especially, main contribution of the $\mathcal{T}$-breaking terms would appear in $\mu \rightarrow e \gamma$.

\subsubsection{Constraint from the $\mu \rightarrow e \gamma$ process}

The $\mu \rightarrow e \gamma$ process has been well investigated in 2 HDMs [76, 77]. The MEG experiment released the upper bound on the branching ratio of the flavor changing process: $\operatorname{Br}(\mu \rightarrow$ $e \gamma)<5.7 \times 10^{-13}$ [78]. It would be updated up to $6 \times 10^{-14}$ in the future [79].

Our dominant contribution to the $\mu \rightarrow e \gamma$ process is from the one-loop correction involving the scalars of $\phi_{2}$, because $\phi_{2}$ has large $(e, \tau)$ and $(\tau, \mu)$ elements of the flavorviolating Yukawa couplings. If the CP-even scalar and CP-odd scalar masses of $\phi_{2}$ are different, the $\mu \rightarrow e \gamma$ process is easily enhanced. The operator to induce the LFV process is estimated as follows at the one-loop level:

$$
\begin{aligned}
\mathcal{L}_{\mu \rightarrow e \gamma} & =e C_{7} \overline{e_{L}} \sigma_{\mu \nu} \mu_{R} F^{\mu \nu}, \\
C_{7} & =\frac{m_{\tau} Y_{e 2}^{\tau} Y_{\mu 2}^{\tau}}{64 \pi^{2}}\left\{\frac{U_{2 \alpha}^{h} U_{2 \alpha}^{h}}{m_{h_{\alpha}}^{2}} F\left(m_{h_{\alpha}}^{2} / m_{\tau}^{2}\right)-\frac{U_{2 \alpha}^{A} U_{2 \alpha}^{A}}{m_{A_{\alpha}}^{2}} F\left(m_{A_{\alpha}}^{2} / m_{\tau}^{2}\right)\right\},
\end{aligned}
$$

where $e$ is the electric charge and $F(x)$ is defined as

$$
F(x)=\ln (x)-\frac{3}{2}
$$

$U_{i j}^{h, A}$ are the diagonalizing matrices for the mass matrices of CP-even and -odd scalars;

$$
\begin{aligned}
\left(\left(U^{h}\right)_{a \alpha} m_{h_{\alpha}}^{2}\left(U^{h}\right)_{b \alpha}\right) & =\left(\begin{array}{cc}
m_{\phi_{2}}^{2}+\left(\delta m_{H}^{2}\right)_{22} & \left(\delta m_{H}^{2}\right)_{23} \\
\left(\delta m_{H}^{2}\right)_{23} & m_{\phi_{3}}^{2}+\left(\delta m_{H}^{2}\right)_{33}
\end{array}\right), \\
\left(\left(U^{A}\right)_{a \alpha} m_{A_{\alpha}}^{2}\left(U^{A}\right)_{b \alpha}\right) & =\left(\begin{array}{cc}
m_{\phi_{2}}^{2}+\left(\delta m_{A}^{2}\right)_{22} & \left(\delta m_{A}^{2}\right)_{23} \\
\left(\delta m_{A}^{2}\right)_{23} & m_{\phi_{3}}^{2}+\left(\delta m_{A}^{2}\right)_{33}
\end{array}\right) .
\end{aligned}
$$

Figure 2 shows the excluded region by the current upper bound on $\mu \rightarrow e \gamma$ process, in the case that only $\left(\delta m_{H}^{2}\right)_{22}$ is nonzero. As we see, the mass difference between the CPeven and CP-odd scalars is severely constrained by the flavor-changing process. If $\phi_{2}$ is below $300 \mathrm{GeV}, \tan \beta$ should be smaller than about 2 . This is quite strong, compared to the bound from $e^{+} e^{-} \rightarrow \tau^{+} \tau^{-}$in figure 1 . We may require large $\tan \beta$, for instance, to enhance the muon anomalous magnetic moment [71,72], but the ratio of the squared mass difference to $m_{\phi_{2}}^{2},\left(\delta m_{H}^{2}\right)_{22} / m_{\phi_{2}}^{2}$, should be much smaller than $O\left(10^{-2}\right)$. 


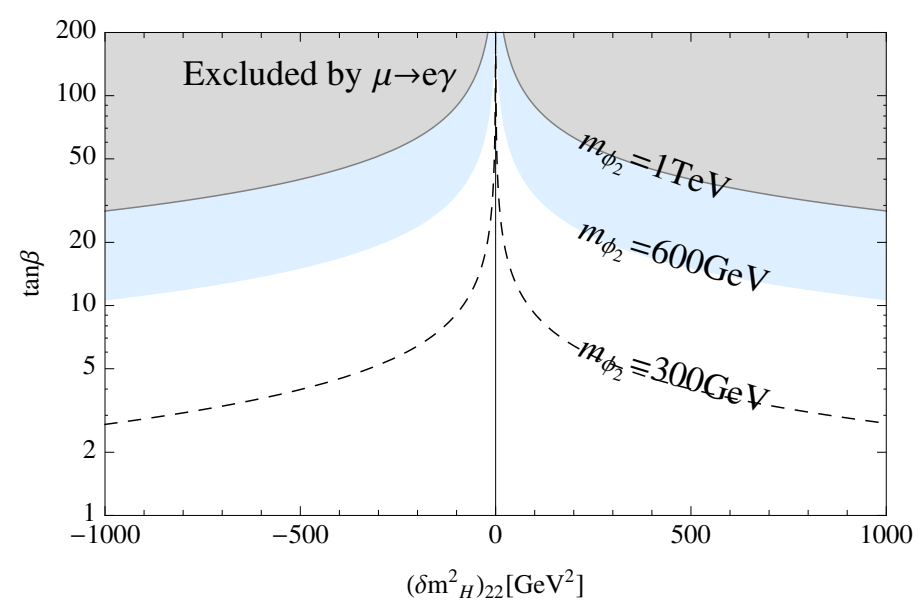

Figure 2. $\left(\delta m_{H}^{2}\right)_{22}(\mathrm{GeV})$ and $\tan \beta$. The gray (light-blue) is excluded by $\mu \rightarrow e \gamma$ at $m_{\phi_{2}}=1000$ (600) $\mathrm{GeV}$. The dashed line is the upper bound with $m_{\phi_{2}}=300 \mathrm{GeV}$.

\subsubsection{Constraint from the $\tau \rightarrow e \gamma$ process}

The scalar of $\phi_{3}$ contribute to the $\tau \rightarrow e \gamma$ process, according to the mass difference between the CP-even and CP-odd scalar. The operator for the LFV process is given by

$$
\begin{aligned}
\mathcal{L}_{\tau \rightarrow e \gamma} & =e C_{7}^{\tau} \overline{e_{L}} \sigma_{\mu \nu} \tau_{R} F^{\mu \nu}, \\
C_{7}^{\tau} & =\frac{m_{\mu} Y_{e 3}^{\mu} Y_{\mu 3}^{\tau}}{64 \pi^{2}}\left\{\frac{U_{3 \alpha}^{h} U_{3 \alpha}^{h}}{m_{h_{\alpha}}^{2}}\left(F\left(m_{h_{\alpha}}^{2} / m_{\mu}^{2}\right)+\frac{m_{\tau}}{6 m_{\mu}}\right)-\frac{U_{3 \alpha}^{A} U_{3 \alpha}^{A}}{m_{A_{\alpha}}^{2}}\left(F\left(m_{A_{\alpha}}^{2} / m_{\mu}^{2}\right)+\frac{m_{\tau}}{6 m_{\mu}}\right)\right\} .
\end{aligned}
$$

The current upper bound on the $\tau \rightarrow e \gamma$ process is $1.1 \times 10^{-7}$ [80], and it is rather weak compared with the one from the $\mu \rightarrow e \gamma$ process. In figure 3, the regions excluded by $\tau \rightarrow e \gamma, \tau^{-} \rightarrow e^{-} \mu^{+} \mu^{-}$, and $\tau^{-} \rightarrow e^{+} \mu^{-} \mu^{-}$are summarized. We can conclude that the $\tau^{-} \rightarrow e^{+} \mu^{-} \mu^{-}$constraint is the most important to $\phi_{3}$ in our model, even if we include the $\mathcal{T}$-breaking terms.

\subsubsection{Muon anomalous magnetic moment $(g-2)_{\mu}$}

In our model, the $\mathcal{T}$ breaking term which allows the mass mixing between $\phi_{2}$ and $\phi_{3}$ enhances the muon anomalous magnetic moment and electron, muon EDMs.

It is well-known that there is a $3.1 \sigma$ deviation from the SM prediction in the muon anomalous magnetic moment $(g-2)_{\mu}$ experimental result [69]. In ref. [71, 72], very light pseudo-scalar is introduced to achieve the anomaly in the leptophilic 2HDM. In our model, we could find new contributions to $(g-2)_{\mu}$ according to the tree-level FCNCs [76],

$$
\Delta a_{\mu}=\frac{m_{\mu} m_{\tau} Y_{\tau 2}^{\mu} Y_{\mu 3}^{\tau}}{(4 \pi)^{2}}\left\{\frac{U_{2 \alpha}^{h} U_{3 \alpha}^{h}}{m_{h_{\alpha}}^{2}} F\left(m_{h_{\alpha}}^{2} / m_{\tau}^{2}\right)-\frac{U_{2 \alpha}^{A} U_{3 \alpha}^{A}}{m_{A_{\alpha}}^{2}} F\left(m_{A_{\alpha}}^{2} / m_{\tau}^{2}\right)\right\} .
$$

Unfortunately, the enhancement of $\Delta a_{\mu}$ is tiny as long as the $\mathcal{T}$-breaking terms are small, because of the stringent constraint from the $\mu \rightarrow e \gamma$ and the $\tau^{-} \rightarrow e^{+} \mu^{-} \mu^{-}$processes. 


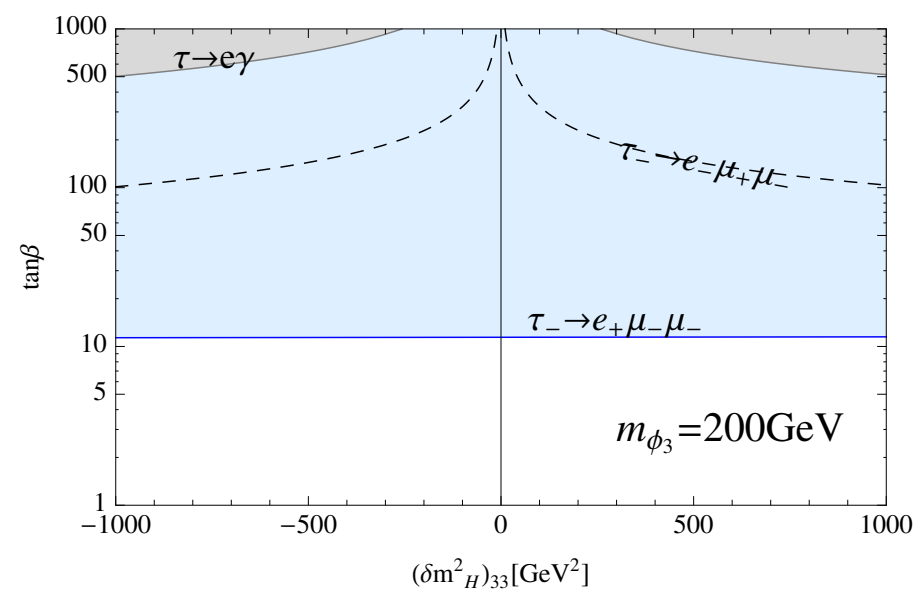

Figure 3. $\left(\delta m_{H}^{2}\right)_{33}(\mathrm{GeV})$ and $\tan \beta$. The gray (light-blue) is excluded by $\tau \rightarrow e \gamma$ at $m_{\phi_{3}}=$ $200 \mathrm{GeV}$. The region above the dashed line is the upper bound from $\tau^{-} \rightarrow e^{-} \mu^{+} \mu^{-}$and the blue region is the excluded one by $\tau^{-} \rightarrow e^{+} \mu^{-} \mu^{-}$.

Setting $\left(\delta m_{H, A}^{2}\right)_{22}=\left(\delta m_{H, A}^{2}\right)_{33}=\left(\delta m_{A}^{2}\right)_{23}=0, \Delta a_{\mu}$ is at most $O\left(10^{-2}\right) \times 10^{-9}$, which is much below the experimental result. One possible way to enhance $\Delta a_{\mu}$ would be large $\tan \beta$ and light $\mathcal{T}$-trivial scalar scenario, as pointed out in ref. [71, 72].

In addition, the loop corrections involving extra scalars with $\mathcal{T}$-breaking terms deviate the mass base of the charged leptons. As long as $\tan \beta$ is rather small, the deviation would be tiny but the large $\tan \beta$ scenario may be also fascinating because of the discrepancy of $(g-2)_{\mu}$. Furthermore, nonzero $\theta_{13}$ is confirmed at the experiments [17-22], so it is important to discuss the contribution of the $\mathcal{T}$-breaking terms to the PMNS matrix. In the next section, we investigate the mass mixing from the one-loop correction, and discuss the contribution to $\theta_{13}$ and the flavor changing processes.

When Yukawa couplings in eq. (4.22) are complex, contributions to electric dipole moment occur from their imaginary parts. The electron and muon EDMs are given as follows:

$$
\begin{aligned}
& d_{e}=\frac{e}{32 \pi^{2}} \operatorname{Im}\left(Y_{e 2}^{\tau} Y_{\tau 3}^{e}\right)\left\{U_{3 \alpha}^{h} U_{2 \alpha}^{h} \frac{m_{\tau}}{m_{h \alpha}^{2}} F\left(m_{h \alpha}^{2} / m_{\tau}^{2}\right)-U_{3 \alpha}^{A} U_{2 \alpha}^{A} \frac{m_{\tau}}{m_{A \alpha}^{2}} F\left(m_{A \alpha}^{2} / m_{\tau}^{2}\right)\right\}, \\
& d_{\mu}=\frac{e}{32 \pi^{2}} \operatorname{Im}\left(Y_{\mu 3}^{\tau} Y_{\tau 2}^{\mu}\right)\left\{U_{3 \alpha}^{h} U_{2 \alpha}^{h} \frac{m_{\tau}}{m_{h \alpha}^{2}} F\left(m_{h \alpha}^{2} / m_{\tau}^{2}\right)-U_{3 \alpha}^{A} U_{2 \alpha}^{A} \frac{m_{\tau}}{m_{A \alpha}^{2}} F\left(m_{A \alpha}^{2} / m_{\tau}^{2}\right)\right\} .
\end{aligned}
$$

The current upper bounds on the electron and muon EDMs are $\left|d_{e}\right|<8.7 \times 10^{-29}[e \mathrm{~cm}][81]$ and $\left|d_{\mu}\right|<1.8 \times 10^{-19}[e \mathrm{~cm}]$ [82], respectively. Figure 4 shows the allowed region in the case with $m_{\phi_{2}}=m_{\phi_{3}}=200 \mathrm{GeV}$. The imaginary parts of $\operatorname{Im}\left(Y_{i 2}^{j} Y_{k 3}^{l}\right)$ are assumed to be given by eqs. (2.11) and (2.19). The red region in figure 4 shows the excluded region by the current experimental bound on the electron EDM. Depending on the phase of the Yukawa couplings, it is the most stringent one among the relevant constraints in our model.

\subsection{Short summary}

Let us summarize the results in this section. We investigate the experimental bounds from flavor physics. In the $\mathcal{T}$-conserving case, the lepton flavor violating decay, $\tau^{-} \rightarrow$ 


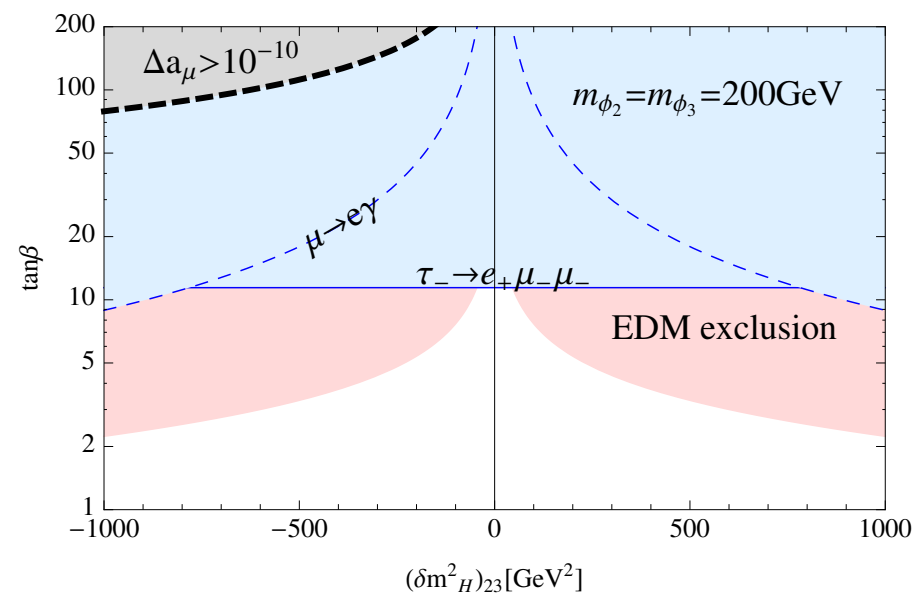

Figure 4. $\left(\delta m_{H}^{2}\right)_{23}(\mathrm{GeV})$ and $\tan \beta$ with $m_{\phi_{2}}=m_{\phi_{3}}=200 \mathrm{GeV}$.

$e^{+} \mu^{-} \mu^{-}$, gives the stringent constraint. If we include the $\mathcal{T}$-breaking terms in the scalar mass matrices, $\mu \rightarrow e \gamma$ and the electron EDM are relevant to our model. We summarize the allowed points in figure 5. $m_{A_{2}}$ and $m_{A_{3}}$ are set to be equal to $m_{\phi}$ and they are within $126 \mathrm{GeV}$ and $1 \mathrm{TeV}$ range. The left figure of figure 5 shows the allowed regions for the $\mathcal{T}$-breaking terms: the blue points correspond to $\left|\left(\delta m_{H}^{2}\right)_{23} / m_{\phi}^{2}\right|$ and $\left|\left(\delta m_{H}^{2}\right)_{22} / m_{\phi}^{2}\right|$ respectively, and the red points figure out $\left|\left(\delta m_{H}^{2}\right)_{33} / m_{\phi}^{2}\right|$. As discussed in this section, $\left(\delta m_{H}^{2}\right)_{22}$ and $\left(\delta m_{H}^{2}\right)_{23}$ are strongly constrained by $\mu \rightarrow e \gamma$ and the electron EDM, while the bound on $\left(\delta m_{H}^{2}\right)_{33}$ is relatively weak. If we $\operatorname{take} \tan \beta$ to be larger than 10 , the $\mathcal{T}$ breaking terms should be less than $O(0.1)$ compared with the $\mathcal{T}$-conserving parts. In other words, the upper bound on $\tan \beta$ is less than 10, if $\left|\left(\delta m_{H}^{2}\right)_{23} / m_{\phi}^{2}\right|$ is larger than $O(0.1)$.

In the right figure of figure 5 , we see the allowed region for $m_{\phi}$ and $\tan \beta .\left|\left(\delta m_{H}^{2}\right)_{a b} / m_{\phi}^{2}\right|$ is larger than 0.01. The black line corresponds to the upper limit with the vanishing $\left(\delta m_{H}^{2}\right)_{a b}$. The red (blue) points are the allowed ones without (with) the constraint from the electron EDM. $Y_{e 2}^{\tau} Y_{\tau 3}^{e}$ is assumed to be pure imaginary on the blue points. As we see, the light $m_{\phi}$ is disfavored by the $\mu \rightarrow e \gamma$ process, while the bound from $\tau^{-} \rightarrow e^{+} \mu^{-} \mu^{-}$is more important in the heavy $m_{\phi}$ region. If $Y_{e 2}^{\tau} Y_{\tau 3}^{e}$ includes imaginary parts, the electron EDM may become more relevant to our model, and give the severe constraint as in figure 5 .

\section{$5 \quad$ Mass mixing induced by $\mathcal{T}$-breaking terms}

Off-diagonal elements of Dirac mass matrix for charged leptons are generated by loop diagrams including interactions coming from $\Delta V$, where the mixing between scalar bosons carrying different $\mathcal{T}$-charges occurs as shown in eq. (4.13). Including corrections for Dirac mass matrix of charged leptons $\left(M_{l}\right)$, let us redefine the mass matrix

$$
M_{l}=\left(\begin{array}{ccc}
m_{e} & \epsilon_{e \mu} & \epsilon_{e \tau} \\
\epsilon_{\mu e} & m_{\mu} & \epsilon_{\mu \tau} \\
\epsilon_{\tau e} & \epsilon_{\tau \mu} & m_{\tau}
\end{array}\right) .
$$



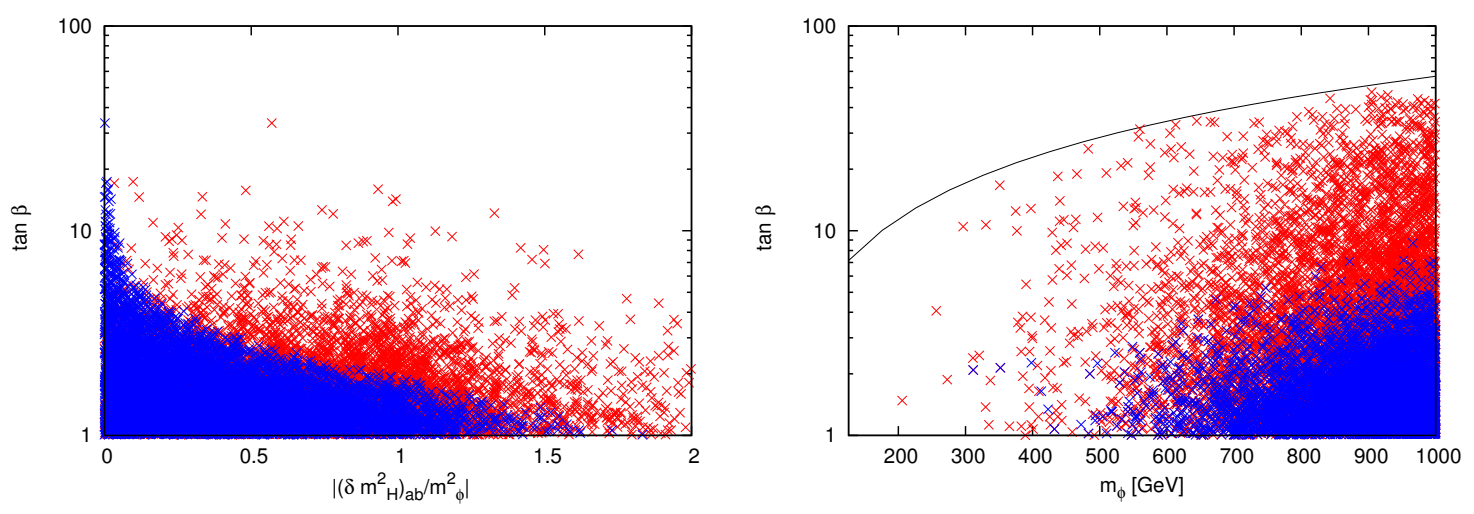

Figure 5. $\left|\left(\delta m_{H}^{2}\right)_{a b} / m_{\phi}^{2}\right|$ and $\tan \beta$ (left), and $m_{\phi}(\mathrm{GeV})$ and $\tan \beta$ (right). $m_{A_{2}}$ and $m_{A_{3}}$ are fixed at $m_{\phi}$, and $m_{\phi}$ is within $126 \mathrm{GeV}$ and $1 \mathrm{TeV}$ range. In the left figure, blue points figure out $\left|\left(\delta m_{H}^{2}\right)_{23} / m_{\phi}^{2}\right|$ and $\left|\left(\delta m_{H}^{2}\right)_{22} / m_{\phi}^{2}\right|$, and red points are $\left(\delta m_{H}^{2}\right)_{33} / m_{\phi}^{2}$. In the right figures, $\left|\left(\delta m_{H}^{2}\right)_{a b} / m_{\phi}^{2}\right|$ is larger than 0.01 . The blue (red) points are the allowed ones for the experimental bounds with (without) the upper limit of the electron EDM. The black line is the upper bound in the case with $\left|\left(\delta m_{H}^{2}\right)_{a b}\right|=0$.

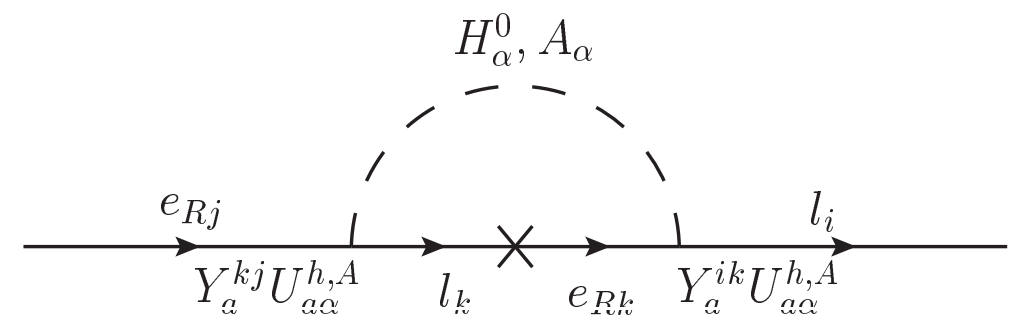

Figure 6. 1-loop diagram which gives $\epsilon_{i j} . H_{a}^{0}, A_{a}$ in this figure is scalar mass eigenstates in $\mathcal{T}$-breaking case.

In order to derive explicit representation for $\epsilon_{i j}$, we consider 1-loop processes as shown in figure 6. The off-diagonal elements of $M_{l}$ are estimated as,

$$
\epsilon_{i j}=\sum_{a, \alpha, \beta} \frac{Y_{i a}^{k} m_{k} Y_{k b}^{j}}{32 \pi^{2}}\left\{U_{a \alpha}^{h} U_{b \alpha}^{h} \ln \frac{m_{h_{\alpha}}^{2}}{\Lambda^{2}}-U_{a \alpha}^{A} U_{b \alpha}^{A} \ln \frac{m_{A_{\alpha}}^{2}}{\Lambda^{2}}\right\}
$$

where $i, j, k$ indices represent charged leptons, and $a, b=2,3$ are defined. $\Lambda$ is some scale, but $\epsilon_{i j}$ do not explicitly depend on $\Lambda$. We assume $m_{k} \ll m_{h_{\alpha}}, m_{A_{\alpha}}$.

These loop corrections change the mass base slightly, and would contribute to the physical observables such as neutrino mixing angles. Here, we investigate how large $\theta_{13}$ can be according to the radiative correction and discuss the correlation between the neutrino mixing and the predicted flavor changing process.

On the other hand, we may find extra FCNCs generated by the radiative corrections. For instance, the Yukawa couplings of the $\mathcal{T}$-trivial scalars are flavor-diagonal at the tree level, but nonzero off-diagonal elements appear at the one-loop level, according to 


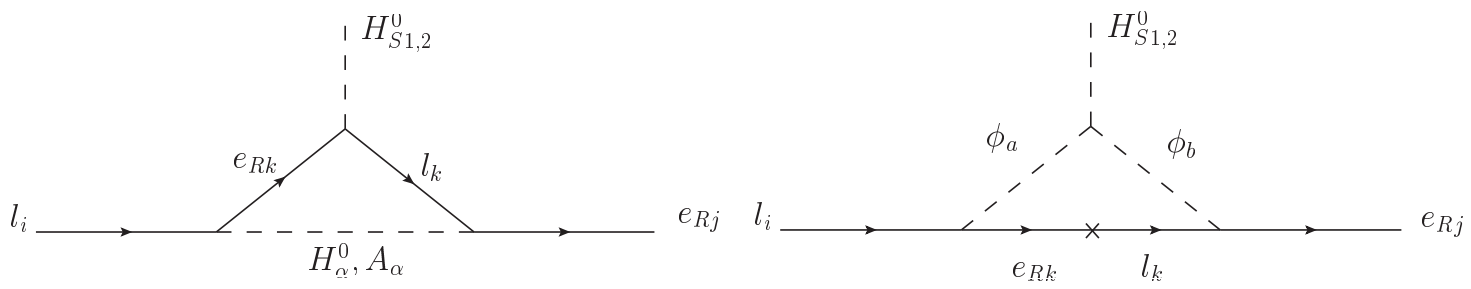

Figure 7. 1-loop processes which result in change of Yukawa couplings. When $\mathcal{T}$-trivial scalar bosons $H_{S 1,2}^{0}$ get VEVs, these processes give mass mixing terms drawn in figure 4.

the radiative correction to the Yukawa couplings involving the $\mathcal{T}$-trivial scalars as shown in figure 7.

The one-loop FCNCs can be described as,

$$
\begin{aligned}
\mathcal{L}_{F C N C}^{(1)} & =-y_{i j}^{1} H_{S 1}^{0} \bar{l}_{i} e_{R j}-y_{i j}^{2} H_{S}^{0} \bar{l}_{i} e_{R j}, \\
y_{i j}^{1} & =\sum_{\alpha, a, b} \frac{Y_{i a}^{k} m_{k} Y_{k b}^{j}}{16 \pi^{2} \sqrt{2}}\left(\cos \alpha \frac{\partial}{\partial\left\langle H_{q}\right\rangle}+\sin \alpha \frac{\partial}{\partial\left\langle H_{1}\right\rangle}\right)\left\{U_{a \alpha}^{h} U_{b \alpha}^{h} \ln \frac{m_{h_{\alpha}}^{2}}{\Lambda^{2}}-U_{a \alpha}^{A} U_{b \alpha}^{A} \ln \frac{m_{A_{\alpha}}^{2}}{\Lambda^{2}}\right\}, \\
y_{i j}^{2} & =\sum_{\alpha, a, b} \frac{Y_{i a}^{k} m_{k} Y_{k b}^{j}}{16 \pi^{2} \sqrt{2}}\left(\cos \alpha \frac{\partial}{\partial\left\langle H_{1}\right\rangle}-\sin \alpha \frac{\partial}{\partial\left\langle H_{q}\right\rangle}\right)\left\{U_{a \alpha}^{h} U_{b \alpha}^{h} \ln \frac{m_{h_{\alpha}}^{2}}{\Lambda^{2}}-U_{a \alpha}^{A} U_{b \alpha}^{A} \ln \frac{m_{A_{\alpha}}^{2}}{\Lambda^{2}}\right\} .
\end{aligned}
$$

These Yukawa couplings are vanishing in the $\mathcal{T}$-conserving limit, so that they are suppressed by the $\mathcal{T}$-breaking terms. We can expect that $(e, \mu)$ and $(e, \tau)$ elements may be enhanced because of the sizable Yukawa couplings, as we have seen in the $\mu \rightarrow e \gamma$ and $\tau \rightarrow e \gamma$ processes. Assuming $\left(\delta m_{H}^{2}\right)_{22}$ is only nonzero among the $\mathcal{T}$-breaking terms, we find that $y_{e \mu}^{1}$ is approximately estimated as,

$$
y_{e \mu}^{1} \approx-\frac{2 \sqrt{2} C_{7}}{1-F\left(m_{\phi_{2}}^{2} / m_{\tau}^{2}\right)} \times\left(\cos \alpha \frac{\partial m_{\phi_{2}}^{2}}{\partial\left\langle H_{q}\right\rangle}+\sin \alpha \frac{\partial m_{\phi_{2}}^{2}}{\partial\left\langle H_{1}\right\rangle}\right),
$$

where the dependence of $\left\langle H_{1}\right\rangle$ and $\left\langle H_{q}\right\rangle$ in $\left(\delta m_{H}^{2}\right)_{22}$ is ignored. Eventually $y_{e \mu}^{1}$ is very tiny, because of the stringent $\mu \rightarrow e \gamma$ constraint. When the last term is around the EW-scale, $y_{e \mu}^{1}$ is at most $O\left(10^{-12}\right) \cdot y_{e \tau}^{1}$ is also small due to the analogy. The bound is weaker, so it could be slightly larger than the $(e, \mu)$ element, but it is at most $O\left(10^{-9}\right)$. The other elements are much smaller, because of the suppression of Yukawa couplings and $\mathcal{T}$-breaking terms.

Contributions to neutrino mixing angles. Based on the above estimation, we investigate the contribution to the LFV, and the observed neutrino mixing.

In many flavor models, the full flavor symmetry is broken to its subgroups, which are different from each other between the charged lepton sector and the neutrino sector. In a certain type of models $\theta_{13}=0$ is predicted at tree level, while other models lead to non-zero $\theta_{13}$. Here, we restrict ourselves to the former case, that is, $\theta_{13}=0$ at the first stage. However, as we disscussed above, T-breaking effects from $\Delta V \neq 0$ may modify the prediction, and neutrino mixing matrix is altered to have non-zero $\theta_{13}$. Now, we study the contributions of the $\mathcal{T}$-breaking terms to the neutrino mixing and discuss the possibility that the observed neutrino mixing is achieved by the diagonalizing matrix of charged leptons. 
$\mathcal{S}$-breaking entering into the neutrino sector also gives non-zero $\theta_{13}$, but this effect highly depends on the setup of neutrino sector; whether the right-handed neutrinos is present or not, how many if there is, whether the seesaw mechanism arises or not, which type of them if it arises, etc.. Thus, we concentrate on the charged lepton sector to give model independent considerations.

The size of correction for the off-diagonal elements of Dirac mass matrix is given in eq. (5.2). In addition, $\epsilon_{i j}$ may be induced by extra heavy particles decoupling at some scale $(\Lambda)$ or small deviations of the vacuum alignment, i.e., $\left\langle H_{2,3}^{0}\right\rangle \neq 0$. Then the diagonalizing matrices $U_{L}, U_{R}$ for charged leptons are corrected to be,

$$
U_{L}^{\dagger} \simeq\left(\begin{array}{ccc}
1 & -\frac{\epsilon_{e \mu}}{m_{\mu}} & -\frac{\epsilon_{e \tau}}{m_{\tau}} \\
\frac{\epsilon_{e \mu}}{m_{\mu}} & 1 & -\frac{\epsilon_{\mu \tau}}{m_{\tau}} \\
\frac{\epsilon_{e \tau}}{m_{\tau}} & \frac{\epsilon_{\mu \tau}}{m_{\tau}} & 1
\end{array}\right), \quad U_{R} \simeq\left(\begin{array}{ccc}
1 & \frac{\epsilon_{\mu e}}{m_{\mu}} & \frac{\epsilon_{\tau e}}{m_{\tau}} \\
-\frac{\epsilon_{\mu e}}{m_{\mu}} & 1 & \frac{\epsilon_{\tau \mu}}{m_{\tau}} \\
-\frac{\epsilon_{\tau e}}{m_{\tau}} & -\frac{\epsilon_{\tau \mu}}{m_{\tau}} & 1
\end{array}\right) .
$$

These small deviations modify the neutrino mixing matrix from the Tri-Bi maximal matrix;

$$
U_{P M N S}=\left(\begin{array}{ccc}
1 & -\frac{\epsilon_{e \mu}}{m_{\mu}} & -\frac{\epsilon_{e \tau}}{m_{\tau}} \\
\frac{\epsilon_{e \mu}}{m_{\mu}} & 1 & -\frac{\epsilon_{\mu \tau}}{m_{\tau}} \\
\frac{\epsilon_{e \tau}}{m_{\tau}} & \frac{\epsilon_{\mu \tau}}{m_{\tau}} & 1
\end{array}\right)\left(\begin{array}{ccc}
\sqrt{\frac{2}{3}} & \frac{1}{\sqrt{3}} & 0 \\
-\frac{1}{\sqrt{6}} & \frac{1}{\sqrt{3}} & -\frac{1}{\sqrt{2}} \\
-\frac{1}{\sqrt{6}} & \frac{1}{\sqrt{3}} & \frac{1}{\sqrt{2}}
\end{array}\right) .
$$

Then $\sin \theta_{13} \sim \epsilon_{e \mu} / \sqrt{2} m_{\mu}$ is generated. If the contribution of the neutrino sector to $\sin \theta_{13}$ is negligible, we find the required value for the observed $\sin \theta_{13}: \epsilon_{e \mu} \approx 0.2 \times m_{\mu}$.

First, let us discuss the one-loop corrections to the neutrino mixings. We can write $\theta_{13}$ in terms of $\mathcal{T}$-breaking effects, according to eq. (5.7):

$$
\begin{aligned}
\sin \theta_{13}^{\text {lepton }} & =\frac{\epsilon_{e \mu}}{\sqrt{2} m_{\mu}}-\frac{\epsilon_{e \tau}}{\sqrt{2} m_{\tau}} \\
& \simeq \frac{Y_{e 2}^{\tau} Y_{\tau 2}^{\mu}}{\sqrt{2}(4 \pi)^{2}} \frac{m_{\tau}}{m_{\mu}}\left(U_{2 \alpha}^{h} U_{2 \alpha}^{h} \ln \frac{m_{h_{\alpha}}^{2}}{m_{\tau}^{2}}-U_{2 \alpha}^{A} U_{2 \alpha}^{A} \ln \frac{m_{A_{\alpha}}^{2}}{m_{\tau}^{2}}\right) .
\end{aligned}
$$

Here, we neglect a term of $\epsilon_{e \tau} / m_{\tau}$, because this term is suppressed by a factor $m_{\mu}^{2} / m_{\tau}^{2}$ as compared with the first term.

$\epsilon_{i j}$ are generated by the $\mathcal{T}$-breaking terms which are strongly constrained by especially $\mu \rightarrow e \gamma$. We see the predicted points on $\theta_{13}^{\text {lepton }}$ allowed by the $\mu \rightarrow e \gamma$ constraint, in figure 8. Note that $\sin \theta_{13}^{\text {lepton }}$ is proportional to $\cos ^{-2} \beta$, so that it is easily enhanced by $\tan \beta$. In figure $8, \tan \beta$ is less than 100 , and then $\sin \theta_{13}$ could be $O(0.01)$. Similary, other shifts of mixing angles from Tri-Bi maximal values are written as

$$
\begin{aligned}
\sin \theta_{12}^{\text {lepton }}-\frac{1}{\sqrt{3}} & \simeq \frac{Y_{e 2}^{\tau} Y_{\tau 2}^{\mu}}{\sqrt{3}(4 \pi)^{2}} \frac{m_{\tau}}{m_{\mu}}\left(U_{2 \alpha}^{h} U_{2 \alpha}^{h} \ln \frac{m_{h_{\alpha}}^{2}}{m_{\tau}^{2}}-U_{2 \alpha}^{A} U_{2 \alpha}^{A} \ln \frac{m_{A_{\alpha}}^{2}}{m_{\tau}^{2}}\right), \\
\sin \theta_{23}^{\text {lepton }}-\left(-\frac{1}{\sqrt{2}}\right) & \simeq \frac{Y_{\mu 2}^{e} Y_{e 2}^{\tau}}{\sqrt{2}(4 \pi)^{2}} \frac{m_{e}}{m_{\tau}}\left(U_{2 \alpha}^{h} U_{2 \alpha}^{h} \ln \frac{m_{h_{\alpha}}^{2}}{m_{e}^{2}}-U_{2 \alpha}^{A} U_{2 \alpha}^{A} \ln \frac{m_{A_{\alpha}}^{2}}{m_{e}^{2}}\right) .
\end{aligned}
$$

$\epsilon_{e \tau}$ in $\sin \theta_{12}$ is neglected as in the case of $\theta_{13}$. A contribution to $\sin \theta_{23}^{\text {lepton }}$ from $\mathcal{T}$-breaking in the charged lepton sector is extremely small because of the factor $m_{e} / m_{\tau} \cdot \sin \theta_{12}^{\text {lepton }}$ 


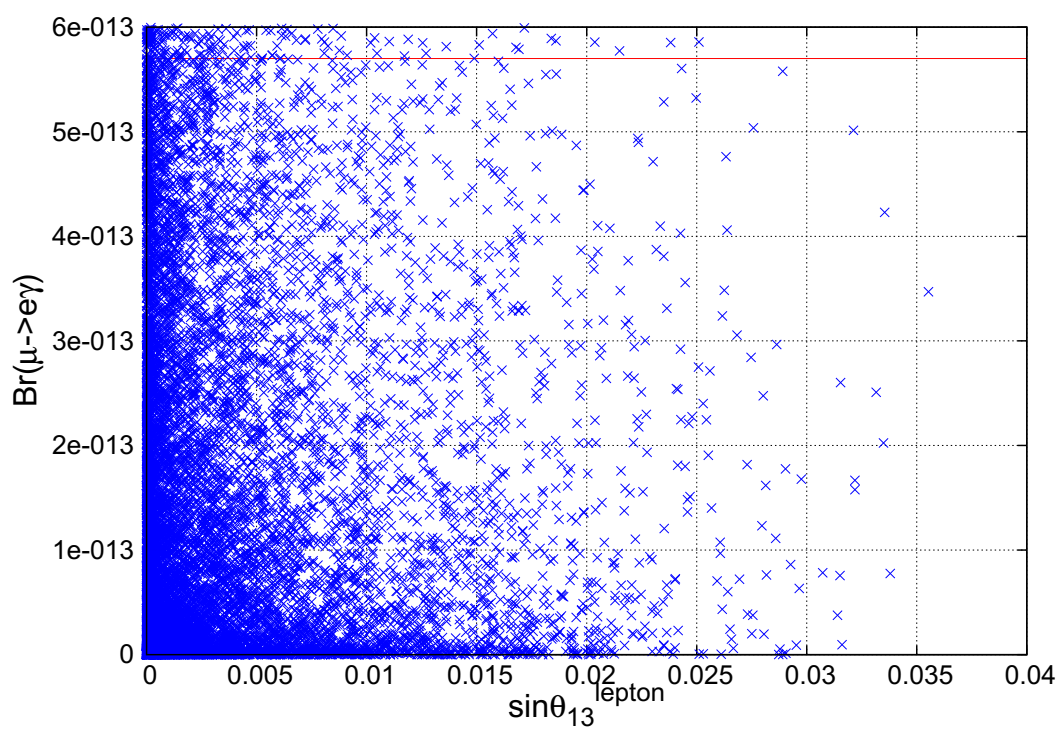

Figure 8. Some of possible values of $\left(\sin \theta_{13}^{\text {lepton }}, \operatorname{Br}(\mu \rightarrow e \gamma)\right)$ for $\tan \beta<100$. The larger $\sin \theta_{13}^{\text {lepton }}$ is, the smaller value of $\operatorname{Br}(\mu \rightarrow e \gamma)$ is tend to favored and vice versa. This is because $\operatorname{Br}(\mu \rightarrow e \gamma)$ is suppressed by factors $\frac{1}{m_{h}^{2}}, \frac{1}{m_{A}^{2}}$ compared with $\sin \theta_{13}^{\text {lepton }}$. The horizontal line shows experimental upper bound of $\operatorname{Br}(\mu \rightarrow e \gamma)$ [78].

correlates with $\operatorname{Br}(\mu \rightarrow e \gamma)$ as same as the case of $\theta_{13}^{\text {lepton }}$. On the other hand, the correction to $\sin \theta_{23}^{\text {lepton }}$ seems to be much smaller than the others.

In the above argument, it is attempted to give $\sin \theta_{13}$ without considering details of neutrino sector which is highly model dependent. However, a contribution to $\sin \theta_{13}$ coming from only loop-induced $\mathcal{T}$-breaking effect on charged lepton mixing may be small compared to the observed value, and then we would like to refer to a possibility of other contributions to $\theta_{13}$ from charged lepton sector.

We can introduce new higher dimensional operators which predict new lepton mixing to supply additional contribution to $\theta_{13}$. Such operators occur when heavy particles coupling with $\mathcal{G}$-charged scalars decouple at some scale. It would be enough to consider additional terms as follows:

$$
\zeta_{a i j} \frac{\Phi_{a}}{\Lambda} H_{q} \overline{l_{L}^{i}} l_{R}^{j}+\text { h.c. }
$$

where $\Lambda$ is the decoupling scale and $\zeta_{i j k}$ is defined by $\mathcal{G}$. Then, mass mixing terms, $\epsilon_{i j}$, are enhanced, according to the nonzero VEVs of $\Phi$ and $H_{q}$. Especially, the term in eq. (5.11) corresponding to $\epsilon_{e \mu}$ adds a new contribution in the form of $\zeta\langle\Phi\rangle\left\langle H_{q}\right\rangle / \sqrt{2} m_{\mu} \Lambda$ to $\sin \theta_{13}^{\text {lepton }}$. Then the size of the coefficient of this effective interaction has to be $\zeta / \Lambda=O\left(10^{-5}\right) \times\langle\Phi\rangle^{-1}$ to realize $\sin \theta_{13}=O(0.1)$.

Secondly, we could consider the possibility that the Higgs VEV alignment is deviated. When the VEV alignment of $H_{a}$ is altered, the remnant symmetry $\mathcal{T}$ is broken and becomes just approximate symmetry even in the charged lepton sector. When $H_{2}^{0}$ and $H_{3}^{0}$ gain 
nonzero VEVs as $\left\langle H_{2,3}^{0}\right\rangle=\delta v_{2,3} / \sqrt{2}$, mass mixing terms in the form of

$$
\left(\epsilon_{2}^{\delta v}\right)_{e \tau} \equiv Y_{e 2}^{\tau} \frac{\delta v_{2}}{\sqrt{2}}=\frac{m_{\tau} \delta v_{2}}{v \cos \beta},\left(\epsilon_{3}^{\delta v}\right)_{e \mu} \equiv Y_{e 3}^{\mu} \frac{\delta v_{3}}{\sqrt{2}}=\frac{m_{\mu} \delta v_{3}}{v \cos \beta}
$$

are added to $\epsilon_{e \tau}$ and $\epsilon_{e \mu}$ respectively. The size of $\delta v_{2,3}$ has to be $O(0.1) \times v \cos \beta$ to realize $\sin \theta_{13}=O(0.1)$. These deviations change the Yukawa couplings for $\phi_{2}$ and $\phi_{3}$ in the base that charged leptons are mass eigenstates. Besides, the mass mixing terms between $\mathcal{T}$-trivial and $\mathcal{T}$-charged scalars can be induced by, for instance, $\left|H_{a}\right|^{2}\left|H_{q}\right|^{2}$ term in the Higgs potential. Let us approximately describe the deviated Yukawa couplings for $\phi_{2}$ or $\phi_{3}$ assuming $\delta v_{2,3} \ll v \cos \beta$ :

$$
\begin{aligned}
\mathcal{L}_{\mathcal{T}}^{\prime} & =-Y_{i 2}^{\prime j} \phi_{2}^{\prime} \bar{l}_{L}^{i} l_{R}^{j}-Y_{i 3}^{\prime j} \phi_{3}^{\prime} \overline{l_{L}^{i}} l_{R}^{j}, \\
Y_{i 2}^{\prime j} & =\left(U_{L}^{\dagger}\right)^{i k} Y_{k 2}^{l}\left(U_{R}\right)^{l j}-\frac{\delta v_{2}}{v \cos \beta} \frac{\sqrt{2} m_{i}}{v \cos \beta} \delta_{i j}, \\
Y_{i 3}^{\prime j} & =\left(U_{L}^{\dagger}\right)^{i k} Y_{k 3}^{l}\left(U_{R}\right)^{l j}-\frac{\delta v_{3}}{v \cos \beta} \frac{\sqrt{2} m_{i}}{v \cos \beta} \delta_{i j} .
\end{aligned}
$$

In these descriptions, we take the SM limit that the SM Higgs around $125 \mathrm{GeV}$ does not have tree-level FCNCs and its Yukawa couplings are the same as the SM ones. Then, the mass bases of $\phi_{2}$ and $\phi_{3}$ are slightly deviated by $\delta v_{2}$ and $\delta v_{3}$. The mass bases of CP-even and CP-odd scalars in $\phi_{2}^{\prime}$ and $\phi_{3}^{\prime}$ may be the same in this limit.

As we have already discussed, the LFV given by $\phi_{3}$ exchanging is dominated by the LFV $\tau$ decay, $\tau^{-} \rightarrow e^{+} \mu^{-} \mu^{-}$, which is the $\mathcal{T}$-conserving process. The extra $\mathcal{T}$-breaking terms in addition to the mass mixing in eq. (4.13) enhance the other LFV $\tau$ decays as follows:

$$
\begin{aligned}
& \operatorname{Br}\left(\tau^{-} \rightarrow e^{-} \mu^{+} \mu^{-}\right) \simeq\left(\frac{\delta v_{2}}{v \cos \beta} \frac{m_{\phi_{3}}^{2}}{m_{\phi_{2}}^{2}}\right)^{2} \times \operatorname{Br}\left(\tau^{-} \rightarrow e^{+} \mu^{-} \mu^{-}\right), \\
& \operatorname{Br}\left(\tau^{-} \rightarrow e^{-} e^{+} \mu^{-}\right) \simeq\left\{\left(\frac{\epsilon_{e \tau}}{m_{\tau}}+\frac{\epsilon_{\tau \mu}}{m_{\mu}}\right) \frac{m_{\phi_{3}}^{2}}{m_{\phi_{2}}^{2}}+\frac{\epsilon_{e \mu}}{m_{\mu}}\right\}^{2} \times \operatorname{Br}\left(\tau^{-} \rightarrow e^{+} \mu^{-} \mu^{-}\right), \\
& \operatorname{Br}\left(\tau^{-} \rightarrow \mu^{-} \mu^{+} \mu^{-}\right) \simeq\left(\frac{\epsilon_{e \mu}-\epsilon_{\tau \mu}}{m_{\mu}}-\frac{\delta v_{3}}{v \cos \beta}\right)^{2} \times \operatorname{Br}\left(\tau^{-} \rightarrow e^{+} \mu^{-} \mu^{-}\right) .
\end{aligned}
$$

Note that $\epsilon_{i j}$ in these equations include the contributions of the loop corrections, the higherdimensional operators in eq. (5.11), and $\left(\epsilon_{2,3}^{\delta v}\right)_{i j}$ in eq. (5.12). The suppression factors in these processes could be estimated as $\sin ^{2} \theta_{13}^{\text {lepton }}$, so that they are predicted around the region with $O\left(10^{-2}\right) \times \operatorname{Br}\left(\tau^{-} \rightarrow e^{+} \mu^{-} \mu^{-}\right)$, which is safe for the current experimental bound as far as the $\tau^{-} \rightarrow e^{+} \mu^{-} \mu^{-}$bound is evaded.

If the CP-even and CP-odd scalars in especially $\phi_{2}^{\prime}$ have different masses, the branching ratio of $\mu \rightarrow e \gamma$ would be as discussed in section 4.2. Moreover, the ones of $\tau \rightarrow e \gamma$ and $\tau \rightarrow \mu \gamma$ would be also enhanced, according to the nonzero $\sin \theta_{13}^{\text {lepton }}$ :

$$
\begin{aligned}
& \operatorname{Br}\left(\tau^{-} \rightarrow e^{-} \gamma\right) \simeq\left(\frac{\epsilon_{e \tau}}{m_{\tau}}-\frac{\delta v_{2}}{v \cos \beta}\right)^{2} \times \frac{m_{\tau}^{2}}{m_{\mu}^{2}} \times \operatorname{Br}\left(\mu^{-} \rightarrow e^{-} \gamma\right), \\
& \operatorname{Br}\left(\tau^{-} \rightarrow \mu^{-} \gamma\right) \simeq\left(\frac{\epsilon_{e \tau}}{m_{\tau}}-\frac{\delta v_{2}}{v \cos \beta}\right)^{2} \times \operatorname{Br}\left(\mu^{-} \rightarrow e^{-} \gamma\right) .
\end{aligned}
$$


Eventually, $\operatorname{Br}\left(\tau^{-} \rightarrow e^{-} \gamma\right)$ is predicted around the region compatible with $\operatorname{Br}\left(\mu^{-} \rightarrow e^{-} \gamma\right)$, and then the constraints from the exotic $\tau$ decay are less serious than the one from $\mu^{-} \rightarrow e^{-} \gamma$.

\section{Summary}

The origin of the flavor structure of the fermions in the SM is one of the mysteries which have been discussed for a long time. The SM gauge groups are orthogonal to the generation, and we can find flavor symmetry rotating the generations, if we ignore the Yukawa couplings to generate the mass matrices for the fermions according to the spontaneous EW symmetry breaking. This fact may suggest the possibility that the flavor symmetry exists at the high energy and then the observed mass matrices are generated dynamically. Besides, we may be able to find some fragments of the flavor symmetry in the SM. The lepton sector especially may still hold some remnant symmetry of the flavor symmetry respected at the high-energy scale. The remnant ones may give some hints for not only model building but also how to prove flavor symmetric models in flavor physics.

In this paper, we investigated flavor physics in models with flavor symmetry, $\mathcal{G}$, in a quite general manner. In our setup, only leptons are charged under $\mathcal{G}$ and extra Higgs doublets are introduced to respect the flavor symmetry in the Yukawa couplings. The Higgs doublets are assumed to belong to non-trivial irreducible representations of $\mathcal{G}$. Then $\mathcal{G}$ is spontaneously broken by VEVs of $\mathcal{G}$-triplet Higgs bosons, $H_{a}$ and $\Phi$. Some remnant symmetry is left after the symmetry breaking: $\mathcal{T}$ is conserved in the charged lepton sector and $\mathcal{S}$ in the neutrino sector respectively. This framework has been used to realize a specific neutrino mixing pattern such as the Bi maximal or the Tri-Bi maximal mixing. The leptophilic $\mathcal{G}$-triplet $H_{a}$ breaks $\mathcal{G}$ to $\mathcal{T}$, and the EW singlet $\Phi_{a}$, which couples only neutrinos, breaks $\mathcal{G}$ to $\mathcal{S}$.

The symmetry $\mathcal{T}$ plays a crucial role in the control of the FCNCs, although it is not respected in the full lagrangian. $\mathcal{T}$-breaking terms would appear in the Higgs potential and neutrino mass matrix, but they could be also under control once we assume the vacuum alignment of $H_{a}$ and $\Phi_{a}$.

In our study, $\mathcal{T}$ is considered as especially $\mathcal{T}=Z_{3}$, and the constraints from the LFV processes are investigated. In the basis in which $\mathcal{T}$-generator is diagonal, charged leptons are mass eigenstates, while details of Higgs potential need to be analyzed to decide mass eigenstates of Higgs bosons. Charged leptons and mass eigenstates of leptophilic Higgs bosons can be classified into trivial and non-trivial $\mathcal{T}$ singlets after the $\mathcal{G}$ symmetry breaking, where the trivial $\mathcal{T}$-singlet Higgs fields only develop the VEVs. Then $\mathcal{T}$ charge conservation constrains the form of interactions involving charged leptons: the Yukawa couplings of charged leptons and scalars are decided by the remnant symmetry. Especially, $\mathcal{T}$-charged Higgs bosons can have FCNCs and cause multi-leptonic decays and flavor nonuniversal gauge couplings.

We considered the scenario that $\tau$ and $\mu$ leptons carry non-trivial $\mathcal{T}$ charges, and then the LFV $\tau$ decay, $\tau^{-} \rightarrow e^{+} \mu^{-} \mu^{-}$, is predicted through the $\mathcal{T}$-charged scalar exchanging. The flavor violating scattering, $e^{+} e^{-} \rightarrow \tau^{+} \tau^{-}$, could be also sizable, so we investigated the 
constraints. The masses of $\mathcal{T}$-charged scalars are expected to be around the EW scale, so we conclude that $\tan \beta$ should be less than $O(10)$.

On the other hand, the neutrinophilic scalar, $\Phi_{a}$, breaks $\mathcal{T}$ in the neutrino sector, and this $\mathcal{T}$ breaking would propagate into the charged lepton sector through the interactions between $H_{a}$ and $\Phi_{a}$ in the Higgs potential. In fact, flavor changing processes that do not conserve $\mathcal{T}$ charges occur at the one-loop level such as $\mu \rightarrow e \gamma$ and $\tau \rightarrow e \gamma$, which are important when the model prediction is compared with the experimental bounds. In addition, the muon anomalous magnetic moment could be enhanced, although the large enhancement is excluded by the bound from the exotic $\tau$ decay. Figures 3 and 4 show that $\mu \rightarrow e \gamma$ and the electron EDM strongly constrain our models if $\mathcal{T}$ breaking terms in the Higgs potential are allowed: $\tan \beta \lesssim 10$. In other words, the observations, as well as $\tau^{-} \rightarrow e^{+} \mu^{-} \mu^{-}$, are the most relevant to our flavor models.

In this type of models argued in this paper, neutrino mixing with $\theta_{13}=0$ tends to be predicted because Bi maximal or Tri-Bi maximal mixing is realized. Then the remnant symmetry breaking effects may be required to alter this mixing pattern. In section 5, we discussed the case that the remnant symmetry $\mathcal{T}$ is slightly broken in the charged lepton sector. As mentioned above, the $\mathcal{T}$ breaking effect caused by VEVs of $\Phi_{a}$ enters into the charged lepton sector through the loop processes involving scalars. However, this contribution is too small to achieve the observed value of $\theta_{13}$. We also considered additional $\mathcal{T}$-breaking effects to realize the large $\theta_{13}$. Such newly added $\mathcal{T}$-breaking terms also contributes to FCNCs, then we pointed out the correlation between $\theta_{13}$ and FCNCs caused by additional $\mathcal{T}$ breaking effect. For instance, the branching ratios of $\tau^{-} \rightarrow e^{-} \mu^{+} \mu^{-}$, $e^{-} e^{+} \mu^{-}$and $\mu^{-} \mu^{+} \mu^{-}$becomes the almost same order as $\operatorname{Br}\left(\tau^{-} \rightarrow e^{+} \mu^{-} \mu^{-}\right)$suppressed by $O\left(10^{-2}\right)$, and $\operatorname{Br}\left(\tau^{-} \rightarrow e^{-} \gamma\right)$ may be the same order as $\operatorname{Br}\left(\mu^{-} \rightarrow e^{-} \gamma\right)$. The process, $\tau^{-} \rightarrow e^{+} \mu^{-} \mu^{-}$, is the most important in our model, but these predictions would be also useful to test our flavor models.

In this study, we take the SM limit, so the SM-like Higgs around $125 \mathrm{GeV}$ does not have FCNCs. As discussed recently in refs. [83-90], it may be interesting to allow the tree-level FCNCs involving the SM-like Higgs, motivated by the CMS excess in the $h \rightarrow \mu \tau$ channel [91] as well as the muon anomalous magnetic moment [83]. However, our model would not enhance the $(g-2)_{\mu}$ because of the chirality structure of the FCNCs, and then the possible way is to consider very light pseudoscalar and large $\tan \beta$ to achieve the discrepancy of $(g-2)_{\mu}[71,72]$. Such a parameter set would require the tuning of the $\mathcal{T}$-breaking terms and large mass differences among the scalars to evade the strong bounds from $\mu \rightarrow e \gamma$, the electron EDM and $\tau^{-} \rightarrow e^{+} \mu^{-} \mu^{-}$.

Note that we studied flavor physics assuming the relation in eq. (2.11) and $\mathcal{T}=Z_{n}$ $(n \neq 2)$. These conditions may be relevant to our prediction, so we will discuss the other possibility such as $\mathcal{T}=Z_{2}$ in the future.

\section{Acknowledgments}

This work is supported by Grant-in-Aid for Scientific research from the Ministry of Education, Science, Sports, and Culture (MEXT), Japan, No. 23104011 (for Y.O.) and No. 25400252 (for T.K.). 


\section{A Mass matrices and vacuum alignment in $\boldsymbol{A}_{4}$ model}

As an illustration of the argument in section 3, I show the most simple example with $\mathcal{G}=A_{4}, \mathcal{T}=Z_{3}, \mathcal{S}=Z_{2}$. In this model, $H_{q}$ is $A_{4}$ singlet, $H=\left(H_{1}, H_{2}, H_{3}\right)$ and $\Phi=\left(\Phi_{1}, \Phi_{2}, \Phi_{3}\right)$ are $A_{4}$ triplets. $H_{q}$ and $H_{a}(a=1,2,3)$ are $\mathrm{SU}(2)_{L}$ doublets, and $\Phi_{a}$ are treated as gauge singlet real scalar bosons for simplisity.

In the base that diagonalize $Z_{3}$ generator $T_{H}, A_{4}$ is generated by,

$$
T_{H}=\left(\begin{array}{ccc}
1 & 0 & 0 \\
0 & \omega & 0 \\
0 & 0 & \omega^{2}
\end{array}\right), \quad S_{\Phi}=\frac{1}{3}\left(\begin{array}{ccc}
-1 & 2 & 2 \\
2 & -1 & 2 \\
2 & 2 & -1
\end{array}\right),
$$

for 3 representation. The generators for 1, 1' and 1" representations of $A_{4}$ are $S_{\Phi}=\mathbf{1}$ and $T_{H}=\mathbf{1}, \omega, \omega^{2}$ respectively.

In the base in which $\mathcal{S}$ generator is diagonal, two generators of $A_{4}$ are written as

$$
\widetilde{S}_{\Phi}=\left(\begin{array}{ccc}
1 & 0 & 0 \\
0 & -1 & 0 \\
0 & 0 & -1
\end{array}\right), \quad \widetilde{T}_{H}=\left(\begin{array}{lll}
0 & 1 & 0 \\
0 & 0 & 1 \\
1 & 0 & 0
\end{array}\right),
$$

for 3 representation. For trivial and non-trivial singlets, these two generators are $\widetilde{S}_{\Phi}=\mathbf{1}$, $\widetilde{T}_{H}=1, \omega, \omega^{2} . \Phi$ is here assumed to be gauge singlet for simplicity. In this basis, the general form of interaction terms between scalar bosons in our $A_{4}$ model, which can give the expected vacuum alignment, ${ }^{5}$ is,

$$
\begin{aligned}
V_{H}\left(H_{q}, H_{a}\right)= & \mu_{q}^{2} H_{q}^{\dagger} H_{q}+\mu^{2}\left\{H_{1}^{\dagger} H_{1}+H_{2}^{\dagger} H_{2}+H_{3}^{\dagger} H_{3}\right\} \\
& +\lambda_{1}\left(H_{q}^{\dagger} H_{q}\right)^{2}+\lambda_{2}\left\{\left(H_{1}^{\dagger} H_{1}\right)^{2}+\left(H_{2}^{\dagger} H_{2}\right)^{2}+\left(H_{3}^{\dagger} H_{3}\right)^{2}\right\} \\
& +\lambda_{3}\left\{\left(H_{1}^{\dagger} H_{1}\right)\left(H_{2}^{\dagger} H_{2}\right)+\left(H_{2}^{\dagger} H_{2}\right)\left(H_{3}^{\dagger} H_{3}\right)+\left(H_{3}^{\dagger} H_{3}\right)\left(H_{1}^{\dagger} H_{1}\right)\right\} \\
& +\lambda_{4}\left\{\left(H_{1}^{\dagger} H_{2}\right)^{2}+\left(H_{2}^{\dagger} H_{3}\right)^{2}+\left(H_{3}^{\dagger} H_{1}\right)^{2}+\text { h.c. }\right\} \\
& +\lambda_{5}\left\{\left|H_{1}^{\dagger} H_{2}\right|^{2}+\left|H_{2}^{\dagger} H_{3}\right|^{2}+\left|H_{3}^{\dagger} H_{1}\right|^{2}\right\} \\
& +\lambda_{6}\left\{\left(H_{1}^{\dagger} H_{1}\right)\left(H_{q}^{\dagger} H_{q}\right)+\left(H_{2}^{\dagger} H_{2}\right)\left(H_{q}^{\dagger} H_{q}\right)+\left(H_{3}^{\dagger} H_{3}\right)\left(H_{q}^{\dagger} H_{q}\right)\right\} \\
& +\lambda_{7}\left\{\left(H_{1}^{\dagger} H_{q}\right)^{2}+\left(H_{2}^{\dagger} H_{q}\right)^{2}+\left(H_{3}^{\dagger} H_{q}\right)^{2}+\text { h.c. }\right\} \\
& +\lambda_{8}\left\{\left|H_{1}^{\dagger} H_{q}\right|^{2}+\left|H_{2}^{\dagger} H_{q}\right|^{2}+\left|H_{3}^{\dagger} H_{q}\right|^{2}\right\} \\
& +\lambda_{9}\left\{\left(H_{1}^{\dagger} H_{2}\right)\left(H_{3}^{\dagger} H_{q}\right)+\left(H_{2}^{\dagger} H_{3}\right)\left(H_{1}^{\dagger} H_{q}\right)+\left(H_{3}^{\dagger} H_{1}\right)\left(H_{2}^{\dagger} H_{q}\right)+\text { h.c. }\right\} \\
& +\lambda_{10}\left\{\left(H_{2}^{\dagger} H_{1}\right)\left(H_{3}^{\dagger} H_{q}\right)+\left(H_{3}^{\dagger} H_{2}\right)\left(H_{1}^{\dagger} H_{q}\right)+\left(H_{1}^{\dagger} H_{3}\right)\left(H_{2}^{\dagger} H_{q}\right)+\text { h.c. }\right\}, \\
& \mu_{\Phi}^{2}\left(\Phi_{1}^{2}+\Phi_{2}^{2}+\Phi_{3}^{2}\right) \\
& +\lambda_{1}^{\Phi}\left(\Phi_{1}^{4}+\Phi_{2}^{4}+\Phi_{3}^{4}\right)+\lambda_{2}^{\Phi}\left(\Phi_{1}^{2} \Phi_{2}^{2}+\Phi_{2}^{2} \Phi_{3}^{2}+\Phi_{3}^{2} \Phi_{1}^{2}\right) \\
& +\lambda_{3}^{\Phi} H_{q}^{\dagger} H_{q}\left(\Phi_{1}^{2}+\Phi_{2}^{2}+\Phi_{3}^{2}\right),
\end{aligned}
$$

\footnotetext{
${ }^{5}$ We do not include three point interaction terms in the Higgs potential below because they cause tadpole terms of $\mathcal{T}$ and $\mathcal{S}$-charged fields which make our VEV alignment unstable.
} 


$$
\begin{aligned}
\Delta V\left(H_{a}, \Phi_{a}\right)= & \lambda_{1}^{\Delta}\left(\Phi_{1}^{2} H_{1}^{\dagger} H_{1}+\Phi_{2}^{2} H_{2}^{\dagger} H_{2}+\Phi_{3}^{2} H_{3}^{\dagger} H_{3}\right) \\
& +\lambda_{2}^{\Delta}\left(\Phi_{1}^{2} H_{2}^{\dagger} H_{2}+\Phi_{2}^{2} H_{3}^{\dagger} H_{3}+\Phi_{3}^{2} H_{1}^{\dagger} H_{1}\right) \\
& +\lambda_{3}^{\Delta}\left(\Phi_{1}^{2} H_{3}^{\dagger} H_{3}+\Phi_{2}^{2} H_{1}^{\dagger} H_{1}+\Phi_{3}^{2} H_{2}^{\dagger} H_{2}\right) \\
& +\lambda_{4}^{\Delta}\left(\Phi_{1} \Phi_{2} H_{1}^{\dagger} H_{2}+\Phi_{2} \Phi_{3} H_{2}^{\dagger} H_{3}+\Phi_{3} \Phi_{1} H_{3}^{\dagger} H_{1}+\text { h.c. }\right)
\end{aligned}
$$

In order to leave $Z_{3}$ in the charged lepton sector and $Z_{2}$ in the neutrino sector, leptophilic boson $H$ and neutrinophilic boson $\Phi$ have to obtain VEVs as

$$
\left(\left\langle H_{1}\right\rangle,\left\langle H_{2}\right\rangle,\left\langle H_{3}\right\rangle\right)=\left(v_{H} / \sqrt{2}, v_{H} / \sqrt{2}, v_{H} / \sqrt{2}\right), \quad\left(\left\langle\Phi_{1}\right\rangle,\left\langle\Phi_{2}\right\rangle,\left\langle\Phi_{3}\right\rangle\right)=\left(v_{\Phi} / \sqrt{2}, 0,0\right) .(\text { A. } 6)
$$

$H_{q}$ also get VEV $v_{q}$ to give quark masses. Thus, we expand eq. (A.5) around the vacuum,

$$
H_{q}=\left(\begin{array}{c}
H_{q}^{+} \\
\frac{1}{\sqrt{2}}\left(v_{q}+H_{q}^{0}+i A_{q}\right)
\end{array}\right), \quad H_{j}=\left(\begin{array}{c}
H_{j}^{+} \\
\frac{1}{\sqrt{2}}\left(v_{H}+H_{j}^{0}+i A_{j}\right)
\end{array}\right) \quad(j=1,2,3)
$$

and $\Phi_{1}=v_{\Phi} / \sqrt{2}+\Phi_{1}^{0}, \Phi_{2,3}=\Phi_{2,3}^{0}$ to derive mass matrix for scalar bosons. We here assume that the effect of $\Delta V$ is negligibly small as noted in section 3 , that is, $\lambda_{j}^{\Delta} \sim 0$. The mass matrices for charged scalar bosons $\left(H_{q}^{+}, H_{1}^{+}, H_{2}^{+}, H_{3}^{+}\right)$and for neutral CP-odd scalar bosons $\left(A_{q}, A_{1}, A_{2}, A_{3}\right)$ in the $S_{\Phi \text {-diagonal base are }}$

$$
M^{2}=\left(\begin{array}{llll}
a & b & b & b \\
b & c & d & d \\
b & d & c & d \\
b & d & d & c
\end{array}\right),
$$

where $a, b, c$ and $d$ are defined as

$$
\begin{aligned}
a & =\mu_{q}^{2}+\lambda_{1} v_{q}^{2}+\frac{3}{2} \lambda_{6} v_{H}^{2}+\lambda_{3}^{\Phi} v_{\Phi}^{2} \\
b & =\frac{1}{2}\left(2 \lambda_{7}+\lambda_{8}\right) v_{q} v_{H}+\frac{1}{2}\left(\lambda_{9}+\lambda_{10}\right) v_{H}^{2} \\
c & =\mu^{2}+\left(\lambda_{2}+\lambda_{3}\right) v_{H}^{2}+\frac{1}{2} \lambda_{6} v_{q}^{2} \\
d & =\frac{1}{2}\left(2 \lambda_{4}+\lambda_{5}\right) v_{H}^{2}+\frac{1}{2}\left(\lambda_{9}+\lambda_{10}\right) v_{q} v_{H}
\end{aligned}
$$

for charged scalars, and

$$
\begin{aligned}
a & =\frac{\mu_{q}^{2}}{2}+\frac{1}{2} \lambda_{1} v_{q}^{2}+\frac{3}{4}\left(\lambda_{6}-2 \lambda_{7}+\lambda_{8}\right) v_{H}^{2}+\frac{\lambda_{3}^{\Phi}}{2} v_{\Phi}^{2}, \\
b & =\lambda_{7} v_{q} v_{H}+\frac{1}{4}\left(\lambda_{9}+\lambda_{10}\right) v_{H}^{2}, \\
c & =\frac{\mu^{2}}{2}+\frac{1}{2}\left(\lambda_{2}+\lambda_{3}-2 \lambda_{4}+\lambda_{5}\right) v_{H}^{2}+\frac{1}{4}\left(\lambda_{6}-2 \lambda_{7}+\lambda_{8}\right) v_{q}^{2}, \\
d & =\lambda_{4} v_{H}^{2}+\frac{1}{4}\left(\lambda_{9}+\lambda_{10}\right) v_{q} v_{H},
\end{aligned}
$$

for neutral CP-odd states. 
The mass matrix for the neutral CP-even scalar bosons $\left(H_{q}^{0}, H_{1}^{0}, H_{2}^{0}, H_{3}^{0}, \Phi_{1}^{0}, \Phi_{2}^{0}, \Phi_{3}^{0}\right)$ in the $S_{\Phi}$-diagonal base is

$$
M^{2}=\left(\begin{array}{c|ccc|ccc}
A & B & B & B & G & 0 & 0 \\
\hline B & C & D & D & & & \\
B & D & C & D & \multicolumn{2}{|c}{0} & \\
B & D & D & C & & & \\
\hline G & & & E & 0 & 0 \\
0 & & 0 & 0 & F & 0 \\
0 & & & 0 & 0 & F
\end{array}\right),
$$

where each element is defined as follows:

$$
\begin{aligned}
A & =\frac{\mu_{q}^{2}}{2}+\frac{3}{2} \lambda_{1} v_{q}^{2}+\frac{3}{4}\left(\lambda_{6}+2 \lambda_{7}+\lambda_{8}\right) v_{H}^{2}+\frac{\lambda_{3}^{\Phi}}{2} v_{\Phi}^{2}, \\
B & =\frac{1}{2}\left(\lambda_{6}+2 \lambda_{7}+\lambda_{8}\right) v_{q} v_{H}+\frac{3}{4}\left(\lambda_{9}+\lambda_{10}\right) v_{H}^{2}, \\
C & =\frac{\mu^{2}}{2}+\frac{1}{2}\left(3 \lambda_{2}+\lambda_{3}+2 \lambda_{4}+\lambda_{5}\right) v_{H}^{2}+\frac{1}{4}\left(\lambda_{6}+2 \lambda_{7}+\lambda_{8}\right) v_{q}^{2}, \\
D & =\frac{1}{2}\left(\lambda_{3}+2 \lambda_{4}+\lambda_{5}\right) v_{H}^{2}+\frac{3}{4}\left(\lambda_{9}+\lambda_{10}\right) v_{q} v_{H}, \\
E & =\frac{\mu_{\Phi}^{2}}{2}+6 \lambda_{1}^{\Phi} v_{\Phi}^{2}+\frac{1}{2} \lambda_{3}^{\Phi} v_{q}^{2} \\
F & =\frac{\mu_{\Phi}^{2}}{2}+\lambda_{2}^{\Phi} v_{\Phi}^{2}+\frac{1}{2} \lambda_{3}^{\Phi} v_{q}^{2} \\
G & =\lambda_{3}^{\Phi} v_{\Phi} v_{q} .
\end{aligned}
$$

In order to move to the basis in which $T_{H}$ is diagonal we rotate $A_{4}$ triplets with

$$
U_{\omega}=\frac{1}{\sqrt{3}}\left(\begin{array}{ccc}
1 & 1 & 1 \\
1 & \omega^{2} & \omega \\
1 & \omega & \omega^{2}
\end{array}\right),
$$

for $U_{\omega} T_{H} U_{\omega}^{\dagger}=\operatorname{diag}\left(1, \omega, \omega^{2}\right)$, which is the form of eq. (2.7), and the VEV is defined as

$$
\sqrt{3} v_{H}=v \cos \beta, \quad v_{q}=v \sin \beta .
$$

All mass submatrices for $A_{4}$-triplet scalar bosons are rotated and then we find mass matrices of the scalars in the form of eq. (3.2).

Nonzero $\Delta V$ causes $\mathcal{T}$-breaking terms as follows:

$$
\begin{aligned}
\delta m^{2}= & \frac{1}{2} \lambda_{1}^{\Delta} v_{\Phi}^{2} H_{1}^{02}+\frac{1}{2} \lambda_{2}^{\Delta} v_{\Phi}^{2} H_{2}^{02}+\frac{1}{2} \lambda_{3}^{\Delta} v_{\Phi}^{2} H_{3}^{02} \\
& +\frac{1}{2} \lambda_{1}^{\Delta} v_{\Phi}^{2} A_{1}^{2}+\frac{1}{2} \lambda_{2}^{\Delta} v_{\Phi}^{2} A_{2}^{2}+\frac{1}{2} \lambda_{3}^{\Delta} v_{\Phi}^{2} A_{3}^{2} \\
& +\lambda_{1}^{\Delta} v_{\Phi}^{2} H_{1}^{-} H_{1}^{+}+\lambda_{2}^{\Delta} v_{\Phi}^{2} H_{2}^{-} H_{2}^{+}+\lambda_{3}^{\Delta} v_{\Phi}^{2} H_{3}^{-} H_{3}^{+} \\
& +\frac{1}{2}\left(\lambda_{1}^{\Delta}+\lambda_{2}^{\Delta}+\lambda_{3}^{\Delta}\right) v_{H}^{2}\left(\Phi_{1}^{02}+\Phi_{2}^{02}+\Phi_{3}^{02}\right)+\lambda_{4}^{\Delta} v_{H}^{2}\left(\Phi_{1}^{0} \Phi_{2}^{0}+\Phi_{2}^{0} \Phi_{3}^{0}+\Phi_{3}^{0} \Phi_{1}^{0}\right) \\
& +2 \lambda_{1}^{\Delta} v_{\Phi} v_{H} H_{1}^{0} \Phi_{1}^{0}+2 \lambda_{2} v_{\Phi} v_{H} H_{2}^{0} \Phi_{1}^{0}+2 \lambda_{3}^{\Delta} v_{\Phi} v_{H} H_{3}^{0} \Phi_{1}^{0} \\
& +\lambda_{4}^{\Delta} v_{\Phi} v_{H}\left(H_{1}^{0} \Phi_{2}^{0}+H_{2}^{0} \Phi_{2}^{0}+H_{1}^{0} \Phi_{3}^{0}+H_{3}^{0} \Phi_{3}^{0}\right) .
\end{aligned}
$$


In addition, we can read out the $\mathcal{T}$ breaking terms in eq. (4.13) for this model from eq. (A.15):

$$
\left(\delta m_{H}^{2}\right)_{a b}=\left(\delta m_{A}^{2}\right)_{a b}=\left(\delta m_{H^{+}}^{2}\right)_{a b}=\lambda_{a}^{\Delta} v_{\Phi}^{2} \delta_{a b},
$$

in the basis of eq. (A.2). After rotated by $U_{\omega}$, mixing terms between states with different $Z_{3}$ charges occur from these $\left(\delta m^{2}\right)_{a b}$. These terms contribute to $\mathcal{T}$-breaking in the charged lepton sector via loop corrections. Further $\mathcal{T}$-breaking terms may be required to describe observed size of non-zero $\theta_{13}$ as we discuss in section 5 .

Open Access. This article is distributed under the terms of the Creative Commons Attribution License (CC-BY 4.0), which permits any use, distribution and reproduction in any medium, provided the original author(s) and source are credited.

\section{References}

[1] S.L. Glashow, J. Iliopoulos and L. Maiani, Weak Interactions with Lepton-Hadron Symmetry, Phys. Rev. D 2 (1970) 1285 [inSPIRE].

[2] G. Altarelli and F. Feruglio, Discrete Flavor Symmetries and Models of Neutrino Mixing, Rev. Mod. Phys. 82 (2010) 2701 [arXiv: 1002.0211] [INSPIRE].

[3] H. Ishimori, T. Kobayashi, H. Ohki, Y. Shimizu, H. Okada and M. Tanimoto, Non-Abelian Discrete Symmetries in Particle Physics, Prog. Theor. Phys. Suppl. 183 (2010) 1 [arXiv: 1003.3552] [INSPIRE].

[4] S.F. King and C. Luhn, Neutrino Mass and Mixing with Discrete Symmetry, Rept. Prog. Phys. 76 (2013) 056201 [arXiv: 1301.1340] [INSPIRE].

[5] P.F. Harrison, D.H. Perkins and W.G. Scott, Tri-bimaximal mixing and the neutrino oscillation data, Phys. Lett. B 530 (2002) 167 [hep-ph/0202074] [INSPIRE].

[6] P.F. Harrison and W.G. Scott, Symmetries and generalizations of tri - bimaximal neutrino mixing, Phys. Lett. B 535 (2002) 163 [hep-ph/0203209] [INSPIRE].

[7] P.F. Harrison and W.G. Scott, Permutation symmetry, tri-bimaximal neutrino mixing and the S3 group characters, Phys. Lett. B 557 (2003) 76 [hep-ph/0302025] [InSPIRE].

[8] E. Ma and G. Rajasekaran, Softly broken $A_{4}$ symmetry for nearly degenerate neutrino masses, Phys. Rev. D 64 (2001) 113012 [hep-ph/0106291] [INSPIRE].

[9] G. Altarelli and F. Feruglio, Tri-bimaximal neutrino mixing, $A_{4}$ and the modular symmetry, Nucl. Phys. B 741 (2006) 215 [hep-ph/0512103] [INSPIRE].

[10] G. Altarelli and D. Meloni, A Simplest $A_{4}$ Model for Tri-Bimaximal Neutrino Mixing, J. Phys. G 36 (2009) 085005 [arXiv:0905.0620] [InSPIRE].

[11] M. Hirsch, S. Morisi, E. Peinado and J.W.F. Valle, Discrete dark matter, Phys. Rev. D 82 (2010) 116003 [arXiv: 1007.0871] [INSPIRE].

[12] M.S. Boucenna, M. Hirsch, S. Morisi, E. Peinado, M. Taoso and J.W.F. Valle, Phenomenology of Dark Matter from $A_{4}$ Flavor Symmetry, JHEP 05 (2011) 037 [arXiv: 1101.2874] [INSPIRE].

[13] C.S. Lam, Symmetry of Lepton Mixing, Phys. Lett. B 656 (2007) 193 [arXiv:0708.3665] [INSPIRE]. 
[14] C.S. Lam, Determining Horizontal Symmetry from Neutrino Mixing, Phys. Rev. Lett. 101 (2008) 121602 [arXiv:0804.2622] [INSPIRE].

[15] C.S. Lam, The Unique Horizontal Symmetry of Leptons, Phys. Rev. D 78 (2008) 073015 [arXiv:0809.1185] [INSPIRE].

[16] I. de Medeiros Varzielas, S.F. King and G.G. Ross, Neutrino tri-bi-maximal mixing from a non-Abelian discrete family symmetry, Phys. Lett. B 648 (2007) 201 [hep-ph/0607045] [INSPIRE].

[17] Daya BAY collaboration, F.P. An et al., Observation of electron-antineutrino disappearance at Daya Bay, Phys. Rev. Lett. 108 (2012) 171803 [arXiv:1203.1669] [INSPIRE].

[18] DaYA BAY collaboration, F.P. An et al., Improved Measurement of Electron Antineutrino Disappearance at Daya Bay, Chin. Phys. C 37 (2013) 011001 [arXiv:1210.6327] [INSPIRE].

[19] Double CHOOZ collaboration, Y. Abe et al., Indication for the disappearance of reactor electron antineutrinos in the Double CHOOZ experiment, Phys. Rev. Lett. 108 (2012) 131801 [arXiv: 1112.6353] [INSPIRE].

[20] RENO collaboration, J.K. Ahn et al., Observation of Reactor Electron Antineutrino Disappearance in the RENO Experiment, Phys. Rev. Lett. 108 (2012) 191802 [arXiv: 1204.0626] [INSPIRE].

[21] T2K collaboration, K. Abe et al., Indication of Electron Neutrino Appearance from an Accelerator-produced Off-axis Muon Neutrino Beam, Phys. Rev. Lett. 107 (2011) 041801 [arXiv: 1106.2822] [INSPIRE].

[22] MINOS collaboration, P. Adamson et al., Improved search for muon-neutrino to electron-neutrino oscillations in MINOS, Phys. Rev. Lett. 107 (2011) 181802 [arXiv:1108.0015] [INSPIRE].

[23] Y. Hamada, T. Kobayashi, A. Ogasahara, Y. Omura, F. Takayama and D. Yasuhara, Revisiting discrete dark matter model: $\theta_{13} \neq 0$ and $\nu_{R}$ dark matter, JHEP 10 (2014) 183 [arXiv: 1405.3592] [INSPIRE].

[24] G. Altarelli, F. Feruglio, L. Merlo and E. Stamou, Discrete Flavour Groups, theta $a_{13}$ and Lepton Flavour Violation, JHEP 08 (2012) 021 [arXiv:1205.4670] [INSPIRE].

[25] Y. Lin, Tri-bimaximal Neutrino Mixing from $A_{4}$ and $\theta_{13} \sim \theta_{C}$, Nucl. Phys. B 824 (2010) 95 [arXiv: 0905.3534] [INSPIRE].

[26] F. Feruglio, C. Hagedorn and R. Ziegler, Lepton Mixing Parameters from Discrete and CP Symmetries, JHEP 07 (2013) 027 [arXiv: 1211.5560] [INSPIRE].

[27] E. Ma, Neutrino Mixing and Geometric CP-violation with $\Delta$ (27) Symmetry, Phys. Lett. B 723 (2013) 161 [arXiv: 1304.1603] [INSPIRE].

[28] H. Ishimori, Y. Shimizu, M. Tanimoto and A. Watanabe, Neutrino masses and mixing from $S_{4}$ flavor twisting, Phys. Rev. D 83 (2011) 033004 [arXiv: 1010.3805] [InSPIRE].

[29] S.F. King and C. Luhn, Trimaximal neutrino mixing from vacuum alignment in $A_{4}$ and $S_{4}$ models, JHEP 09 (2011) 042 [arXiv:1107.5332] [INSPIRE].

[30] R. Krishnan, P.F. Harrison and W.G. Scott, Simplest Neutrino Mixing from $S_{4}$ Symmetry, JHEP 04 (2013) 087 [arXiv: 1211.2000] [INSPIRE].

[31] Y. Grossman and W.H. Ng, Nonzero $\theta_{13}$ in $\mathrm{SO}(3) \rightarrow A_{4}$ lepton models, Phys. Rev. D 91 (2015) 073005 [arXiv: 1404.1413] [INSPIRE]. 
[32] F. Feruglio, C. Hagedorn and R. Ziegler, A realistic pattern of lepton mixing and masses from $S_{4}$ and $C P$, Eur. Phys. J. C 74 (2014) 2753 [arXiv:1303.7178] [INSPIRE].

[33] T. Araki and Y.F. Li, $Q_{6}$ flavor symmetry model for the extension of the minimal standard model by three right-handed sterile neutrinos, Phys. Rev. D 85 (2012) 065016 [arXiv:1112.5819] [INSPIRE].

[34] S. Dev, R.R. Gautam and L. Singh, Broken $S_{3}$ Symmetry in the Neutrino Mass Matrix and Non-Zero $\theta_{13}$, Phys. Lett. B 708 (2012) 284 [arXiv:1201.3755] [INSPIRE].

[35] P.S. Bhupal Dev, B. Dutta, R.N. Mohapatra and M. Severson, $\theta_{13}$ and Proton Decay in a Minimal $\mathrm{SO}(10) \times S_{4}$ model of Flavor, Phys. Rev. D 86 (2012) 035002 [arXiv:1202.4012] [INSPIRE].

[36] I.K. Cooper, S.F. King and C. Luhn, $A_{4} \times$ SU(5) SUSY GUT of Flavour with Trimaximal Neutrino Mixing, JHEP 06 (2012) 130 [arXiv: 1203.1324] [INSPIRE].

[37] Y.H. Ahn and S.K. Kang, Non-zero $\theta_{13}$ and CP-violation in a model with $A_{4}$ flavor symmetry, Phys. Rev. D 86 (2012) 093003 [arXiv: 1203.4185] [INSPIRE].

[38] I. de Medeiros Varzielas and G.G. Ross, Discrete family symmetry, Higgs mediators and theta $_{13}$, JHEP 12 (2012) 041 [arXiv:1203.6636] [INSPIRE].

[39] G. Altarelli, F. Feruglio and L. Merlo, Tri-Bimaximal Neutrino Mixing and Discrete Flavour Symmetries, Fortsch. Phys. 61 (2013) 507 [arXiv:1205.5133] [InSPIRE].

[40] M.-C. Chen, J. Huang, J.-M. O'Bryan, A.M. Wijangco and F. Yu, Compatibility of $\theta_{13}$ and the Type I Seesaw Model with $A_{4}$ Symmetry, JHEP 02 (2013) 021 [arXiv:1210.6982] [INSPIRE].

[41] C. Luhn, Trimaximal $T M_{1}$ neutrino mixing in $S_{4}$ with spontaneous CP-violation, Nucl. Phys. B 875 (2013) 80 [arXiv: 1306.2358] [INSPIRE].

[42] G.-J. Ding and S.F. King, Generalized $C P$ and $\Delta(96)$ family symmetry, Phys. Rev. D 89 (2014) 093020 [arXiv:1403.5846] [INSPIRE].

[43] G.-J. Ding and Y.-L. Zhou, Lepton mixing parameters from $\Delta(48)$ family symmetry and generalised CP, JHEP 06 (2014) 023 [arXiv: 1404.0592] [INSPIRE].

[44] S.F. King and C. Luhn, On the origin of neutrino flavour symmetry, JHEP 10 (2009) 093 [arXiv: 0908.1897] [INSPIRE].

[45] Q.-H. Cao, S. Khalil, E. Ma and H. Okada, Observable $T_{7}$ Lepton Flavor Symmetry at the Large Hadron Collider, Phys. Rev. Lett. 106 (2011) 131801 [arXiv:1009.5415] [INSPIRE].

[46] C. Luhn, K.M. Parattu and A. Wingerter, A Minimal Model of Neutrino Flavor, JHEP 12 (2012) 096 [arXiv:1210.1197] [INSPIRE].

[47] E. Ma, $A_{4}$ symmetry and neutrinos with very different masses, Phys. Rev. D 70 (2004) 031901 [hep-ph/0404199] [INSPIRE].

[48] A.E. Carcamo Hernandez, I. de Medeiros Varzielas, S.G. Kovalenko, H. Päs and I. Schmidt, Lepton masses and mixings in an $A_{4}$ multi-Higgs model with a radiative seesaw mechanism, Phys. Rev. D 88 (2013) 076014 [arXiv:1307.6499] [inSPIRE].

[49] M. Holthausen, M. Lindner and M.A. Schmidt, Lepton flavor at the electroweak scale: A complete $A_{4}$ model, Phys. Rev. D 87 (2013) 033006 [arXiv:1211.5143] [InSPIRE].

[50] G. Altarelli, F. Feruglio and L. Merlo, Revisiting Bimaximal Neutrino Mixing in a Model with $S_{4}$ Discrete Symmetry, JHEP 05 (2009) 020 [arXiv:0903.1940] [INSPIRE]. 
[51] A. Di Iura, C. Hagedorn and D. Meloni, Lepton mixing from the interplay of the alternating group $A_{5}$ and CP, JHEP 08 (2015) 037 [arXiv: 1503.04140] [INSPIRE].

[52] P. Ballett, S. Pascoli and J. Turner, Mixing angle and phase correlations from A5 with generalised CP and their prospects for discovery, arXiv:1503.07543 [INSPIRE].

[53] R.d.A. Toorop, F. Feruglio and C. Hagedorn, Discrete Flavour Symmetries in Light of T2K, Phys. Lett. B 703 (2011) 447 [arXiv:1107.3486] [INSPIRE].

[54] R. de Adelhart Toorop, F. Feruglio and C. Hagedorn, Finite Modular Groups and Lepton Mixing, Nucl. Phys. B 858 (2012) 437 [arXiv:1112.1340] [INSPIRE].

[55] G.-J. Ding, TFH Mixing Patterns, Large $\theta_{13}$ and $\Delta(96)$ Flavor Symmetry, Nucl. Phys. B 862 (2012) 1 [arXiv:1201.3279] [INSPIRE].

[56] S.F. King, C. Luhn and A.J. Stuart, A Grand $\Delta(96) \times \mathrm{SU}(5)$ Flavour Model, Nucl. Phys. B 867 (2013) 203 [arXiv: 1207.5741] [INSPIRE].

[57] C.S. Lam, Finite Symmetry of Leptonic Mass Matrices, Phys. Rev. D 87 (2013) 013001 [arXiv: 1208.5527] [INSPIRE].

[58] M. Holthausen, K.S. Lim and M. Lindner, Lepton Mixing Patterns from a Scan of Finite Discrete Groups, Phys. Lett. B 721 (2013) 61 [arXiv:1212.2411] [INSPIRE].

[59] C.S. Lam, Horizontal symmetries $\Delta(150)$ and $\Delta(600)$, Phys. Rev. D 87 (2013) 053012 [arXiv: 1301.1736] [INSPIRE].

[60] S.F. King, T. Neder and A.J. Stuart, Lepton mixing predictions from $\Delta\left(6 n^{2}\right)$ family Symmetry, Phys. Lett. B 726 (2013) 312 [arXiv:1305.3200] [InSPIRE].

[61] M. Aoki, S. Kanemura, K. Tsumura and K. Yagyu, Models of Yukawa interaction in the two Higgs doublet model and their collider phenomenology, Phys. Rev. D 80 (2009) 015017 [arXiv: 0902.4665] [INSPIRE].

[62] G.C. Branco, P.M. Ferreira, L. Lavoura, M.N. Rebelo, M. Sher and J.P. Silva, Theory and phenomenology of two-Higgs-doublet models, Phys. Rept. 516 (2012) 1 [arXiv:1106.0034] [INSPIRE].

[63] T. Kobayashi, Y. Omura and K. Yoshioka, Flavor Symmetry Breaking and Vacuum Alignment on Orbifolds, Phys. Rev. D 78 (2008) 115006 [arXiv:0809.3064] [INSPIRE].

[64] LEP, DELPHI, OPAL, ALEPH and L3 collaborations, G. Abbiendi et al., Search for Charged Higgs bosons: Combined Results Using LEP Data, Eur. Phys. J. C 73 (2013) 2463 [arXiv:1301.6065] [INSPIRE].

[65] Sld Electroweak Group, Sld Heavy Flavor Group, Delphi, LeP, AlePh, OPAL, LEP Electroweak Working Group and L3 collaborations, A combination of preliminary electroweak measurements and constraints on the standard model, hep-ex/0312023 [INSPIRE].

[66] K. Hayasaka et al., Search for Lepton Flavor Violating Tau Decays into Three Leptons with 719 Million Produced $\tau^{+} \tau^{-}$Pairs, Phys. Lett. B 687 (2010) 139 [arXiv:1001.3221] [INSPIRE].

[67] SINDRUM collaboration, U. Bellgardt et al., Search for the Decay $\mu^{+} \rightarrow e^{+} e^{+} e^{-}$, Nucl. Phys. B 299 (1988) 1 [INSPIRE]. 
[68] A. Crivellin, A. Kokulu and C. Greub, Flavor-phenomenology of two-Higgs-doublet models with generic Yukawa structure, Phys. Rev. D 87 (2013) 094031 [arXiv:1303.5877] [INSPIRE].

[69] Particle Data Group collaboration, K.A. Olive et al., Review of Particle Physics, Chin. Phys. C 38 (2014) 090001 [inSPIRE].

[70] A. Pich, Precision Tau Physics, Prog. Part. Nucl. Phys. 75 (2014) 41 [arXiv:1310.7922] [INSPIRE].

[71] A. Broggio, E.J. Chun, M. Passera, K.M. Patel and S.K. Vempati, Limiting two-Higgs-doublet models, JHEP 11 (2014) 058 [arXiv:1409.3199] [INSPIRE].

[72] T. Abe, R. Sato and K. Yagyu, Lepton-specific two Higgs doublet model as a solution of muon g - 2 anomaly, JHEP 07 (2015) 064 [arXiv: 1504.07059] [INSPIRE].

[73] ATLAS collaboration, Search for charged Higgs bosons decaying via $H^{ \pm} \rightarrow \tau^{ \pm} \nu$ in fully hadronic final states using pp collision data at $\sqrt{s}=8 \mathrm{TeV}$ with the ATLAS detector, JHEP 03 (2015) 088 [arXiv: 1412.6663] [INSPIRE].

[74] CMS collaboration, Search for charged Higgs bosons with the H+ to tau nu decay channel in the fully hadronic final state at sqrt $s=8 \mathrm{TeV}$, CMS-PAS-HIG-14-020 (2014).

[75] T. Hermann, M. Misiak and M. Steinhauser, $\bar{B} \rightarrow X_{s} \gamma$ in the Two Higgs Doublet Model up to Next-to-Next-to-Leading Order in QCD, JHEP 11 (2012) 036 [arXiv:1208.2788] [INSPIRE].

[76] S. Davidson and G.J. Grenier, Lepton flavour violating Higgs and $\tau \rightarrow \mu \gamma$, Phys. Rev. D 81 (2010) 095016 [arXiv: 1001.0434$]$ [INSPIRE].

[77] D. Chang, W.S. Hou and W.-Y. Keung, Two loop contributions of flavor changing neutral Higgs bosons to $\mu \rightarrow e \gamma$, Phys. Rev. D 48 (1993) 217 [hep-ph/9302267] [INSPIRE].

[78] MEG collaboration, J. Adam et al., New constraint on the existence of the $\mu^{+} \rightarrow e^{+} \gamma$ decay, Phys. Rev. Lett. 110 (2013) 201801 [arXiv:1303.0754] [INSPIRE].

[79] A.M. Baldini et al., MEG Upgrade Proposal, arXiv:1301.7225 [INSPIRE].

[80] BABAR collaboration, B. Aubert et al., Searches for Lepton Flavor Violation in the Decays $\tau^{ \pm} \rightarrow e^{ \pm} \gamma$ and $\tau^{ \pm} \rightarrow \mu^{ \pm} \gamma$, Phys. Rev. Lett. 104 (2010) 021802 [arXiv:0908.2381] [INSPIRE].

[81] ACME collaboration, J. Baron et al., Order of Magnitude Smaller Limit on the Electric Dipole Moment of the Electron, Science 343 (2014) 269 [arXiv:1310.7534] [INSPIRE].

[82] Muon (G-2) collaboration, G.W. Bennett et al., An Improved Limit on the Muon Electric Dipole Moment, Phys. Rev. D 80 (2009) 052008 [arXiv:0811.1207] [INSPIRE].

[83] Y. Omura, E. Senaha and K. Tobe, Lepton-flavor-violating Higgs decay $h \rightarrow \mu \tau$ and muon anomalous magnetic moment in a general two Higgs doublet model, JHEP 05 (2015) 028 [arXiv: 1502.07824] [INSPIRE].

[84] D. Aristizabal Sierra and A. Vicente, Explaining the CMS Higgs flavor violating decay excess, Phys. Rev. D 90 (2014) 115004 [arXiv:1409.7690] [INSPIRE].

[85] J. Heeck, M. Holthausen, W. Rodejohann and Y. Shimizu, Higgs $\rightarrow \mu \tau$ in Abelian and non-Abelian flavor symmetry models, Nucl. Phys. B 896 (2015) 281 [arXiv:1412.3671] [INSPIRE].

[86] A. Crivellin, G. D'Ambrosio and J. Heeck, Explaining $h \rightarrow \mu^{ \pm} \tau^{\mp}, B \rightarrow K^{*} \mu^{+} \mu^{-}$and $B \rightarrow K \mu^{+} \mu^{-} / B \rightarrow K e^{+} e^{-}$in a two-Higgs-doublet model with gauged $L_{\mu}-L_{\tau}$, Phys. Rev. Lett. 114 (2015) 151801 [arXiv: 1501.00993] [INSPIRE]. 
[87] L. de Lima, C.S. Machado, R.D. Matheus and L.A.F. do Prado, Higgs Flavor Violation as a Signal to Discriminate Models, arXiv:1501.06923 [INSPIRE].

[88] A. Crivellin, G. D'Ambrosio and J. Heeck, Addressing the LHC flavor anomalies with horizontal gauge symmetries, Phys. Rev. D 91 (2015) 075006 [arXiv:1503.03477] [INSPIRE].

[89] D. Das and A. Kundu, Two hidden scalars around $125 \mathrm{GeV}$ and $h \rightarrow \mu \tau$, Phys. Rev. D 92 (2015) 015009 [arXiv: 1504.01125] [INSPIRE].

[90] B. Bhattacherjee, S. Chakraborty and S. Mukherjee, $H \rightarrow \tau \mu$ and excess in $t \bar{t} H:$ Connecting the dots in the hope for the first glimpse of BSM Higgs signal, arXiv:1505.02688 [INSPIRE].

[91] CMS collaboration, Search for lepton-flavour-violating decays of the Higgs boson, Phys. Lett. B 749 (2015) 337 [arXiv: 1502.07400] [INSPIRE]. 